\title{
L'impact de la littoralisation sur les marchés fonciers Une approche comparative des côtes basque et charentaise
}

\author{
Jeanne Dachary-Bernard*, Frédéric Gaschet**, Sandrine Lyser*, \\ Guillaume Pouyanne**, Stéphane Virol**
}

Le foncier fait l'objet d'une concurrence importante entre différents usages, notamment l'agriculture et le résidentiel. Les territoires littoraux, en fort développement et générateurs d'aménités spécifiques, sont particulièrement sujets à ce type de conflits d'usage. En effet, les aménités littorales exercent une pression sur les prix fonciers, participant à modifier la structure du territoire de manière complémentaire et/ou substituable aux aménités centrales qui traduisent les avantages à la proximité urbaine. Cet article a pour objet d'étudier l'influence des aménités littorales sur la formation des prix en identifiant la variation des valeurs foncières en fonction de la distance au littoral. Pour ce faire, nous procédons à une analyse comparée sur deux espaces littoraux : le littoral charentais - encore fortement agricole, et la côte basque - au degré d'urbanisation plus avancé. Cette comparaison empirique nécessite de mobiliser une méthodologie commune. La méthode des prix hédoniques est développée de manière originale en proposant de mettre en œuvre successivement une estimation semi-paramétrique, puis une modélisation fragmentée, en tenant compte du phénomène de dépendance spatiale. Nous montrons ainsi que la littoralisation s'accompagne, sur la bande littorale, d'une capitalisation importante de la proximité au littoral dans les prix fonciers résidentiels, liée à la présence d'aménités, qui se prolonge vers l'arrière-pays, dans une logique d'accessibilité. La littoralisation perturbe également les marchés fonciers agricoles, y compris rétro-littoraux. La comparaison des deux sites suggère une forte différenciation de l'impact de la littoralisation en fonction des caractéristiques territoriales, qui structurent des aménités plutôt résidentielles sur la côte basque, et davantage agricoles sur la côte charentaise.

\footnotetext{
* UR ADBX - IRSTEA, Bordeaux

** GREThA-UMR 5113-Université de Bordeaux

Les auteurs remercient les deux rapporteurs anonymes pour la qualité de leurs suggestions et de leurs commentaires sur les précédentes versions de l'article ; la Safer Aquitaine-Atlantique pour la mise à disposition à titre gracieux des données pour le Pays basque. Ces travaux s'inscrivent dans les recherches menées au sein des projets ANR Jeune Chercheur «DyFU », PSDR 3 «ACTeR », et Liteau 3 "Respireau ».
} 
$\mathbf{L}$ a Loi littoral a obtenu un satisfecit lors de son bilan de 2006 (Diact, 2007) : elle aurait ainsi «permis d'amortir l'ampleur $d u$ recul des espaces naturels, sans pour autant empêcher nos régions littorales de connaître un développement économique » (1). Parce qu'elle a «posé des principes précurseurs qui gardent aujourd'hui toute leur actualité » (2), "l'heure n'est pas au lancement d'un chantier législatif mais à un approfondissement des principes de la loi ». Ce bilan positif est cependant loin d'être unanime : selon un rapport récent du CGPC (3), « la pression démographique continue que connaissent ces territoires [littoraux] depuis 30 ans du fait de leur attractivité, n'a pas généré la mise en ouvre de politiques globales à l'échelle des difficultés rencontrées (...). » (Fareniaux et Verhlac, 2008). Laissée aux collectivités locales, la régulation de l'espace littoral apparaît «éclatée, pas unifiée », et « la carence des politiques foncières rend pour l'instant difficilement maîtrisables les évolutions en cours. \(Datar, 2004). Si l'intensité des conflits d'usage liés à la littoralisation est régulièrement soulignée, les mécanismes par lesquels s'exerce la pression urbaine méritent d'être approfondis. L'invocation des politiques foncières et de leurs carences dans la maîtrise de l'urbanisation littorale, récemment matérialisée par la multiplication d'établissements publics fonciers locaux dans ces espaces, doit contribuer à orienter la réflexion vers une meilleure connaissance du fonctionnement des marchés fonciers littoraux.

Ainsi, la question de l'influence du littoral sur les prix fonciers prend de plus en plus d'importance à mesure que s'en précisent les enjeux. Partant du constat d'une littérature lacunaire sur la relation entre la distance au littoral et les prix du sol, cet article explore la structuration spatiale des marchés fonciers résidentiels et agricoles en zone littorale. Il compare deux sites aux caractéristiques d'urbanisation côtière différenciées : un espace littoral déjà fortement artificialisé, voire saturé, la côte basque, et un espace encore agricole, mais en voie d'artificialisation rapide, le littoral charentais. Une revue des fondements théoriques de la formation de la valeur foncière sur ces marchés est en premier lieu proposée, ainsi qu'une justification des sites d'étude choisis. Par la suite, la méthode d'investigation de la structuration spatiale des marchés fonciers littoraux retenue, qui combine des techniques non paramétriques et de l'économétrie spatiale, est exposée. Finalement, les principaux résultats obtenus au regard des enjeux théoriques identifiés sont discutés.

\section{Littoralisation et pression foncière : conflits d'usage et aménités sur les espaces côtiers}

Le littoral est soumis à une pression considérable du fait de son attractivité. Le phénomène de littoralisation se caractérise par un puissant mouvement de migration vers les territoires littoraux : des taux de croissance démographique plus élevés que sur le reste du territoire ; une construction de logements d'autant plus dynamique qu'elle est alimentée par le développement des résidences secondaires ; une forte artificialisation des sols alimentée par le modèle du logement individuel, le tout créant, à l'instar des zones périurbaines des grandes villes, un «modèle de développement extrêmement consommateur d'espace» (Datar, 2004). Or, par opposition aux territoires périurbains, le milieu littoral présente la caractéristique d'être restreint. La limitation de l'offre foncière littorale est d'abord naturelle, liée à sa forme spécifique : il s'agit d'une " bande » littorale, où l'espace est rare. Mais la limitation de l'offre foncière trouve aussi sa source dans la réglementation des usages du sol et les dispositifs de protection des espaces fragiles tels que le Conservatoire du littoral ou la Loi littoral. Au niveau local, les documents de planification du type schéma de cohérence territoriale (SCoT) ou plan local d'urbanisme (PLU) accordent une place de plus en plus importante à la protection d'espaces naturels littoraux très convoités. Ces zonages produisent un accroissement des prix fonciers par deux canaux distincts (Lecat, 2006) : un effet rareté qui restreint ainsi l'offre foncière ; un effet aménité ensuite, puisque le maintien à l'état naturel de certains espaces produit des aménités localisées qui sont intégrées dans le prix (Irwin et Bockstael, 2004 ; Geoghegan, 2002).

La limitation de l'offre foncière littorale, combinée à une demande très dynamique, produit des distorsions tant sur le marché immobilier que sur le marché foncier. Sur le marché immobilier, l'accroissement des prix a créé des distorsions sur le marché du logement : certains segments, comme le logement social, le locatif intermédiaire, ou encore la primo-accession y sont notoirement sous-représentés (Cete, 2007).

\footnotetext{
1. Discours du Premier Ministre F. Fillon à l'occasion du Conseil National du Littoral, 31.I.2008, en ligne.

2. Communiqué de presse de J.-L. Borloo, Ministre d'État, ministre de l'Écologie, du Développement et de l'Aménagement Durables, et de N. Kosciusko-Morizet, Secrétaire d'Etat chargée de l'Ecologie, 11.10.2007, en ligne.

3. CGPC : Conseil général des ponts et chaussées.
} 
Sur le marché foncier, l'accroissement des prix induit une forte pression à la conversion résidentielle des terrains agricoles, en vertu du principe de comparaison des rendements futurs entre les usages du sol (Segerson et al., 2006). Ainsi, les prix des terres agricoles atteignent souvent une valeur sans lien direct avec les résultats économiques de l'agriculture. La conséquence est un recul de l'activité agricole, mesuré par une diminution des SAU (Cete, 2007).

Les aménités littorales sont variées et localisées, et la valeur attribuée à ces aménités par les individus va conduire à leur capitalisation dans les prix du foncier. Cependant, ces aménités ne seront pas capitalisées de la même manière suivant l'usage final qui sera fait du sol. Il faut pour cela revenir sur les logiques de formation des valeurs foncières.

Le mécanisme de formation des prix fonciers repose sur le calcul économique des rendements du sol, et dépend donc de l'usage final qui en est fait. Le prix d'une terre agricole est ainsi égal à la valeur actualisée des rendements futurs liés à cette activité, eux-mêmes déterminés sur les marchés internationaux des denrées. Dans l'éventualité d'une conversion d'un usage agricole à un usage urbain, le prix foncier est égal à la somme actualisée des rendements agricoles jusqu'à la date de conversion, et des rendements urbains (nets des coûts) à partir de la date de conversion (Capozza et Helsley, 1989) : le prix du sol intègre donc une composante « urbaine », qui sert de courroie de transmission entre le marché foncier et le marché immobilier.

Il s'avère donc indispensable de différencier le segment du foncier agricole d'une part, et le segment du foncier à bâtir de l'autre, car le mécanisme de formation de la valeur y est fondamentalement différent. En théorie, le segment du foncier agricole peut être considéré comme un marché foncier « pur ", où le sol s'échange uniquement en tant que bien de production; le segment du foncier à bâtir apparaît plus comme un marché hybride, où s'échangent non seulement le sol, mais aussi les droits qui lui sont rattachés, en l'espèce un droit à bâtir.

Sur le segment du foncier à bâtir, la composante urbaine intégrée dans le prix du sol révèle la porosité entre les marchés immobilier et foncier. La logique de transmission de la valeur s'opère de l'immobilier vers le foncier à travers le mécanisme du compte à rebours : l'opérateur définit un prix de vente du bien immobilier (« prix de sortie »), lui soustrait ses coûts (de construction, financiers, etc.) ainsi que sa marge, et définit une charge foncière acceptable, c'est-à-dire un prix du sol maximal qui assure une rentabilité à l'opération. Par construction, le compte à rebours est à l'origine d'un « effet de levier » : les variations des prix immobiliers sont amplifiées sur le marché foncier (Granelle, 1998). La composante urbaine dans le prix foncier est égale à la valeur actualisée de l'augmentation de prix anticipée qui découle de la conversion du terrain en usage urbain (Cavailhès et Wavresky, 2003). C'est une " prime de croissance », qui est d'autant plus élevée que la pression urbaine est forte, et qui peut être extrêmement élevée. Plantinga et al. (2002) estiment qu'aux ÉtatsUnis, la prime de croissance due aux anticipations de conversion dépasse $80 \%$ du prix du sol dans les États les plus urbanisés. Une approche comparable adoptée par Géniaux et Napoleone (2005) conclut que la valeur résidentielle anticipée a un pouvoir explicatif très puissant sur le prix du foncier agricole, soulignant l'importance des anticipations dans la formation de la valeur.

Si l'on accepte la distinction stricte entre un foncier agricole déconnecté du marché immobilier, et un foncier à bâtir étroitement dépendant du marché immobilier, alors l'influence de la proximité au littoral ne doit être sensible que sur le segment des terrains à bâtir. En effet, les aménités procurées par le littoral sont essentiellement des aménités résidentielles (paysagères, récréatives, etc.) ; au contraire, la proximité au littoral peut être source de "désaménités » pour l'activité agricole (salinisation des terres, risques d'inondation, etc.). Notre hypothèse de départ est donc que l'influence de la proximité au littoral est seulement sensible sur le segment des terrains à bâtir, mais négligeable sur le segment des terres agricoles.

L'estimation d'une valeur globale pour la proximité au littoral est l'objet d'une littérature assez importante, mais limitée au marché immobilier. L'enjeu de cette littérature est d'estimer une prime associée aux aménités littorales selon deux approches complémentaires. L'exposition directe au littoral, d'une part, permet de bénéficier d'aménités récréatives, mais aussi d'aménités paysagères (vue sur mer) : les primes vont de $42 \%$ du prix du bien dans le Delaware (Parsons et Noailly, 2004) à $131 \%$ en Caroline du Sud (Pompe, 2008) pour une situation en front de mer. De manière plus précise, Bin et al. (2008) et Ashton et al. (2009) estiment le consentement à payer du « degré de vue sur mer marginal » à respectivement 995 dollars US en Caroline du 
Nord, et 1334 dollars US en Floride. La proximité au littoral, d'autre part, est capitalisée dans les valeurs immobilières. La prime associée à la proximité au littoral décroît avec la distance, à l'instar d'un gradient spatial de prix traditionnel : on retrouve la compensation, traditionnelle en microéconomie spatiale, entre accessibilité et prix du sol. Brown et Polliakowski (1976) estiment que ce gradient est fort : $75 \%$ de la prime est déjà perdue à un kilomètre du littoral. Pour le Finistère, Travers et al. (2008) montrent bien que la prime de rapprochement du littoral décroît sensiblement avec la distance.

L'hypothèse d'une séparation stricte entre les segments du foncier agricole et du foncier à bâtir mérite toutefois d'être nuancée, notamment dans des espaces sous pression comme l'espace littoral. Les anticipations sur les plusvalues de conversion rendent poreux les deux segments, car même une terre échangée comme bien agricole est susceptible de changer d'usage ultérieurement. Dans une étude sur l'ensemble des Comtés américains, Plantinga et al. (2002) montrent qu'un accroissement de la densité résidentielle environnante augmente très sensiblement les prix agricoles. Aussi, même le prix du foncier agricole peut-il intégrer une prime de croissance. La seule différence est qu'elle sera probablement plus faible que pour un terrain à bâtir, pour deux raisons : d'abord, parce que la perception des plus-values est plus éloignée dans le temps, ce qui réduit la valeur actuelle des rendements espérés (Capozza et Li, 1994); ensuite, parce que l'incertitude sur les prix est d'autant plus élevée que la date de conversion est éloignée (Capozza et Helsley, 1990 ; Cunningham, 2006).

Le degré de porosité entre les deux segments du marché foncier peut être considérablement diminué lorsqu'un terrain est déclaré inconstructible, à la suite de dispositions réglementaires nationales ou locales. Le droit à bâtir disparaît, faisant s'évanouir la composante urbaine dans le prix foncier. Dès lors, les valeurs foncières sont a priori insensibles à la proximité au littoral. Cavailhès et Wavresky (2003) postulent ainsi que le prix des terrains non constructibles est insensible à la distance au centre. Cette proposition est confirmée par l'analyse empirique de Mori (1998) pour Amsterdam, qui obtient un gradient de prix foncier plat.

Cependant, ce raisonnement n'est valable qu'à court terme. Tout zonage est révisable, et l'on peut spéculer sur le fait qu'un terrain classé aujourd'hui comme inconstructible devienne constructible demain. La valeur du foncier « encore inconstructible » va alors objectivement résulter d'un calcul probabiliste sur le délai de transformation de la réglementation, sachant que tous les acquéreurs n'ont pas les mêmes « chances » d'obtenir cette transformation (Comby, 1995). C'est pourquoi Geniaux et Napoleone (2005) proposent de modifier le modèle de Capozza et Helsley (1990), en supposant que les acheteurs ne décident pas d'une date de conversion optimale, mais attachent une probabilité de conversion à chaque bien foncier.

$\mathrm{Au}$ terme de cette discussion deux hypothèses centrales sont retenues pour guider l'approche empirique. Il s'agit d'abord d'analyser la capitalisation des aménités littorales dans les valeurs foncières résidentielles comme le suggère la théorie, et de déterminer le cas échéant la forme du gradient de prix résidentiel : cette capitalisation est-elle confinée à la bande côtière ou se propage-t-elle dans l'arrière-pays ? La seconde interrogation concerne l'existence d'un impact de la littoralisation sur les marchés fonciers agricoles, et donc l'existence d'une porosité entre ces derniers et les marchés fonciers résidentiels. Ces deux hypothèses gagnent à être testées, de manière comparative, sur deux terrains aux caractéristiques d'urbanisation côtière très différenciées.

Deux territoires ont été retenus dans le cadre de cette étude des facteurs de pression foncière sur les espaces littoraux (cf. carte) : la CharenteMaritime et le Pays basque. La zone d'étude sélectionnée sur le littoral charentais s'étend sur 283 communes situées, le long du littoral entre la Rochelle et Royan, et à l'intérieur des terres jusqu'à une quarantaine de kilomètres (Saintes). La côte basque comprend 82 communes distribuées en zone côtière entre le sud des Landes (Seignosse) et la frontière espagnole (Hendaye). L'analyse des formes dominantes d'occupation du sol sur la période 2000-2006, conduite ciaprès à partir de Corine Land Cover (4), permet de souligner la différenciation des enjeux de pression foncière sur ces deux territoires.

\footnotetext{
4. La base de données géographiques Corine Land Cover est produite dans le cadre du programme européen de coordination de l'information sur l'environnement Corine, piloté par l'Agence européenne pour l'environnement. II s'agit d'une base vectorielle des usages biophysiques du sol ventilés selon une nomenclature en 44 postes, établie par interprétation d'images satellites. La version utilisée dans cet article est la version 2006, utilisant un seuil de description de 25 ha pour les bases d'états d'usage du sol (cf. graphique I) et de 5 ha pour la base de changements d'usages du sol (cf. tableau 2).
} 
La pression démographique s'est intensifiée sur les deux territoires entre 1999 et 2006, s'établissant à un niveau nettement supérieur à la moyenne nationale $(+0,7 \%$ par an). La croissance démographique est plus élevée sur le littoral basque, déjà sensiblement plus dense, soulignant ainsi l'intensité de la pression résidentielle sur ce territoire (cf. tableau 1). La bande littorale est en moyenne deux fois plus artificialisée sur la côte basque jusqu'à $20 \mathrm{~km}$ de la côte. L'artificialisation décroît continûment sur ce territoire à mesure que l'on s'éloigne du trait de côte, tandis que le littoral charentais fait apparaître un léger « rebond » entre 20 et $30 \mathrm{~km}$ lié à la présence de Saintes (cf. graphique I). L'accessibilité au littoral structure clairement l'artificialisation de la côte basque, qui atteint des niveaux très élevés dans la bande côtière des cinq kilomètres $(35 \%$ de la surface est urbanisée) et décroît très rapidement à mesure de l'éloignement.

Le conflit entre urbanisation potentielle et agriculture apparaît particulièrement prononcé sur le littoral charentais : l'agriculture représente $57 \%$ des usages du sol sur la bande côtière des cinq kilomètres (contre $22 \%$ sur la côte basque), coexistant avec une emprise importante des zones humides sur la bande littorale (cf. graphique I). Au-delà de cinq kilomètres, l'usage agricole est largement dominant, supérieur à $80 \%$ jusqu'à $30 \mathrm{~km}$. Sur la

Tableau 1

Présentation comparée des deux territoires d'étude

\begin{tabular}{|c|c|c|}
\hline & Côte basque & Littoral charentais \\
\hline Nombre de communes & 82 & 243 \\
\hline Population (2006) & 307532 & 493600 \\
\hline $\begin{array}{l}\text { Taux de croissance démographique annuel moyen } \\
(1999-2006) \text { en \% }\end{array}$ & 1,27 & 1,16 \\
\hline $\begin{array}{l}\text { Taux de croissance démographique annuel moyen } \\
(1990-1999) \text { en \% }\end{array}$ & 0,94 & 0,80 \\
\hline Densité démographique hab. / km² (2006) & 178,3 & 126,8 \\
\hline
\end{tabular}

Champ : ensemble des communes des aires d'étude.

Source : RGP, Insee, calcul des auteurs.

\section{Carte}

Localisation et structure des deux territoires d'études : la côte basque entre Seignosse et Hendaye et le littoral charentais entre la Rochelle et Royan

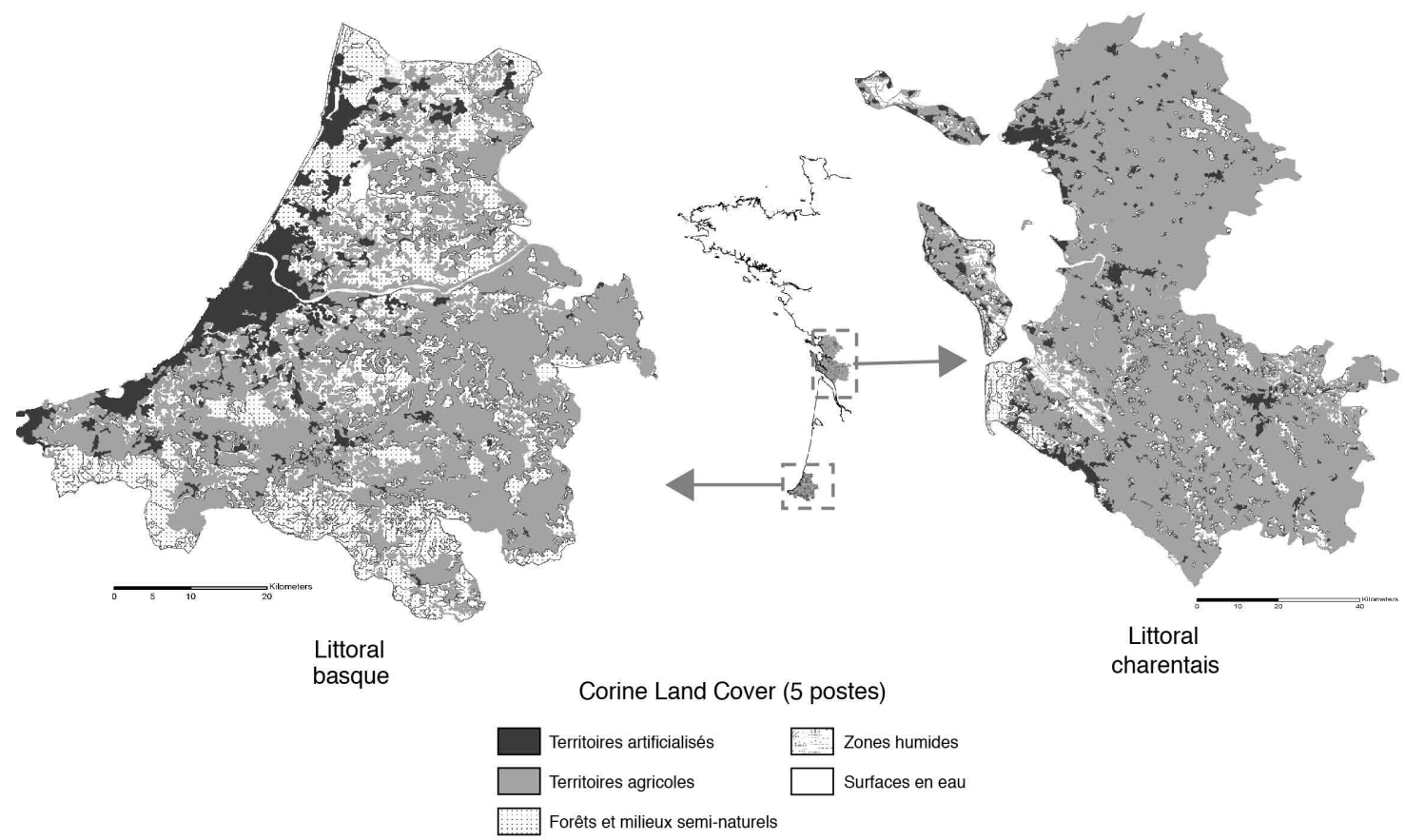


côte basque l'urbanisation, déjà plus avancée, entre en conflit essentiellement avec l'emprise des espaces naturels (la forêt, notamment) ; il semble que l'artificialisation des sols se soit faite principalement au détriment de terres auparavant agricoles, qui sont davantage renvoyées vers l'intérieur des terres : l'emprise agricole n'est nettement dominante qu'au-delà de $20 \mathrm{~km}$.

L'analyse de l'artificialisation récente des sols, intervenue entre 2000 et 2006, fait apparaître un phénomène prononcé de saturation du littoral basque. L'artificialisation récente est particulièrement faible sur la bande côtière (taux inférieur à $2 \%$ jusqu'à $10 \mathrm{~km}$ ) ; elle est reportée sur les espaces entre 10 et $20 \mathrm{~km}$ du littoral (cf. tableau 2).

Le taux d'artificialisation récente de la bande côtière apparaît beaucoup plus marqué sur le littoral charentais, signe de l'ampleur d'un phénomène de rattrapage. La fenêtre de mutation des usages du sol est également plus large sur cet espace et s'étend jusqu'à $30 \mathrm{~km}$. L'urbanisation récente s'est effectuée essentiellement par l'artificialisation de parcelles agricoles (95\% des surfaces concernées), tandis que sur la côte basque, l'artificialisation récente s'est faite principalement en gagnant sur les espaces naturels (40\% des surfaces concernées).

Cette analyse de la dynamique des usages des sols souligne donc une forte différenciation des enjeux de pression foncière des deux espaces. Le littoral charentais combine un degré d'artifi- cialisation encore faible mais en fort développement sur la période récente, la pression de l'urbanisation récente s'exerçant prioritairement au détriment de l'agriculture côtière. La côte basque présente en revanche les caractéristiques d'un espace littoral déjà largement artificialisé par l'urbanisation côtière, en voie de saturation sur la bande littorale, et générant des phénomènes de report de l'urbanisation en retrait de cette bande.

\section{Tableau 2 \\ Changements comparés d'usage du sol entre 2000 et 2006}

\begin{tabular}{|l|c|c|}
\hline $\begin{array}{c}\text { Distance } \\
\text { par rapport } \\
\text { au trait de côte }\end{array}$ & \multicolumn{2}{c|}{$\begin{array}{c}\text { Taux d'urbanisation récente } \\
\text { des territoires artificialisés en 2006 }\end{array}$} \\
\hline C-5 km & Côte basque & Littoral charentais \\
\hline $5-10 \mathrm{~km}$ & 1,74 & 3,05 \\
\hline $10-15 \mathrm{~km}$ & 1,95 & 7,65 \\
\hline $15-20 \mathrm{~km}$ & 8,43 & 8,04 \\
\hline $20-25 \mathrm{~km}$ & 4,60 & 9,37 \\
\hline $25-30 \mathrm{~km}$ & 1,64 & 4,25 \\
\hline $30-40 \mathrm{~km}$ & 0,00 & 5,83 \\
\hline $40-50 \mathrm{~km}$ & 11,53 & 1,51 \\
\hline Total & - & 0,00 \\
\hline
\end{tabular}

Lecture : Il s'agit de la proportion des territoires artificialisés en 2006 qui ont fait l'objet d'une artificialisation entre 2000 et 2006, c'est-à-dire d'une mutation de l'usage dominant du sol. Par exemple sur la Côte basque, pour la plage [0;5 km[ 1,74 \% des territoires artificialisés en 2006 ne l'étaient pas en 2000.

Champ : ensemble des communes des aires d'étude.

Source : Corine Land Cover changes, changements d'usages du sol 2000-2006, révisée selon un seuil de description de 5 ha, traitement et calculs des auteurs.

\section{Graphique I \\ Usages dominants du sol en 2006}

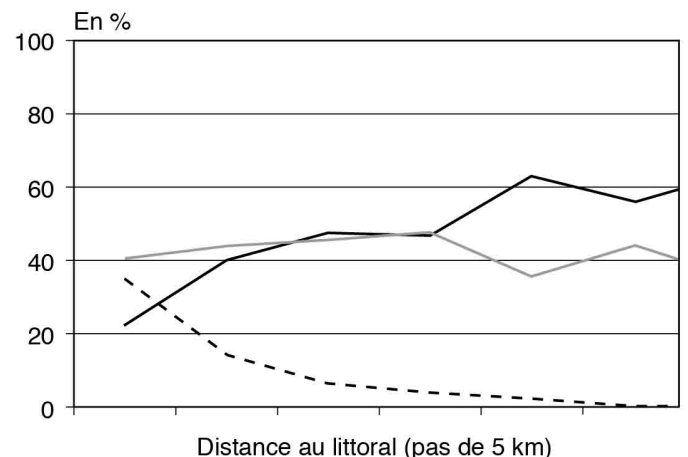

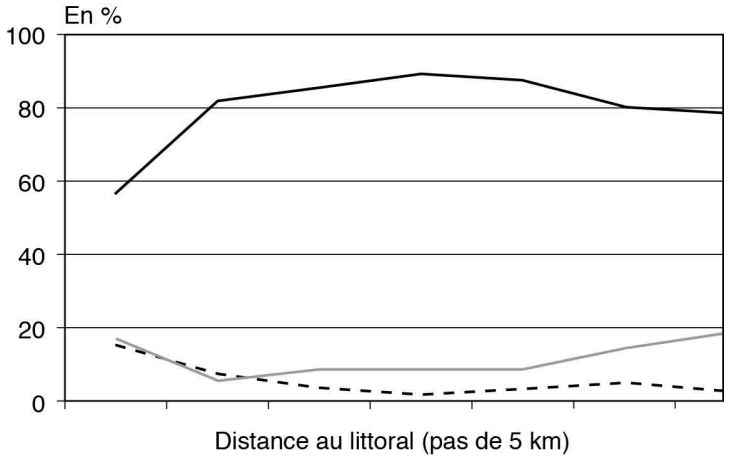

Forêt et espaces semi-naturels

Lecture : la proportion de chaque usage des sols s'obtient à partir du découpage de l'aire d'étude en bandes de $5 \mathrm{~km}$ en partant du trait de côte, et du traitement géomatique des données vectorielles de Corine Land Cover.

Champ : ensemble des usages du sol vectorisés.

Source : Corine Land Cover, 2006. Traitement et calculs des auteurs. 


\section{Valorisation des aménités littorales : méthode hédonique d'estimation des gradients de valeurs foncières}

Les données mobilisées sont issues des notifications collectées par les Sociétés d'aménagement foncier et d'établissement rural (Safer, cf. encadré 1) sur les deux zones littorales présentées précédemment. Ces données correspondent aux transactions réalisées sur la période temporelle considérée, et sont segmentées selon le marché foncier (i.e. selon l'usage de la terre annoncé). Ainsi, nous distinguons le marché foncier agricole (terres et prés) des marchés de type « résidentiel » que sont le marché de l'artificialisation et le marché des maisons à la campagne. Les marchés « des cultures spéciales », « des landes, friches et étangs », « des vignes », « forestier », « des espaces de loisirs » et " hors segmentation», pourtant fournis par les bases, ont été écartés de l'étude pour simplification de l'analyse. Par ailleurs, compte tenu des erreurs d'enregistrement possibles il est apparu nécessaire d'épurer les données brutes, en excluant toutes les transactions se trouvant dans les queues de distribution $(5 \%)$ des prix déflatés et de la surface (Le Goffe et Salanié 2005) (cf. tableau 3).

Tableau 3

Échantillons retenus et prix moyens

\begin{tabular}{|l|c|c|c|c|}
\hline \multirow{2}{*}{ Marchés fonciers } & \multicolumn{2}{|c|}{ Côte basque } & \multicolumn{2}{c|}{ Littoral charentais } \\
\cline { 2 - 5 } & $\begin{array}{c}\text { Nombre } \\
\text { de transactions }\end{array}$ & $\begin{array}{c}\text { Prix moyen } \\
\text { déflaté } € / \text { ha }\end{array}$ & $\begin{array}{c}\text { Nombre } \\
\text { de transactions }\end{array}$ & $\begin{array}{c}\text { Prix moyen } \\
\text { déflaté } € / \text { ha }\end{array}$ \\
\hline Terres et prés & 467 & 26482 & 3119 & 12723 \\
Artificialisation & 933 & 396031 & 2721 & 257753 \\
Maisons à la campagne & 656 & 622643 & 1621 & 791951 \\
\hline Ensemble & 2056 & 384395 & 7461 & 271382 \\
\hline Période d'observation & \multicolumn{2}{|c|}{$2002-2009$} & \multicolumn{2}{|c|}{$2003-2006$} \\
\hline
\end{tabular}

Lecture : nombre et prix moyen (en euros) déflaté par ha des transactions effectuées sur les différents marchés considérés.

Champ : ensemble des transactions Safer échantillonnées par segment de marché et par terrain d'étude.

Source : base Safer, traitement et calculs des auteurs.

Encadré 1

\section{LES BASES DES TRANSACTIONS FONCIÈRES DES SAFER}

Les données des Sociétés d'aménagement foncier et d'établissement rural (Safer) sont issues des déclarations d'intentions d'aliéner (DIA) adressées par les notaires à la Safer et des ventes réalisées par cette dernière. En vertu du Code rural et dans le cadre du droit de préemption de la Safer, les notaires sont tenus d'adresser à cette dernière des notifications de projets de ventes portant sur l'ensemble du marché relatif aux espaces agricoles et naturels. Tous les biens notifiés à la Safer ont donc comme point commun une vocation agricole ou naturelle, qu'elle soit potentielle ou réelle. Ces données correspondent à des ventes de terrains de surface supérieure à un hectare pour lesquelles une vingtaine de variables sont renseignées : prix moyen, caractéristiques de l'acheteur et du vendeur, présence, etc. Certaines transactions inférieures à un hectare peuvent également être contenues dans la base soit parce que le seuil de préemption est inférieur, soit parce que les Safer ont convaincu les notaires d'enregistrer les parcelles plus petites.

Les transactions ont été géolocalisées à deux échelles, en fonction de la disponibilité des informations cadastrales :

- dans le cas où les informations cadastrales (numéro de section et de parcelle) sont renseignées, les transactions sont géolocalisées à la parcelle cadastrale, en s'assurant, en cas de transactions incluant la vente simultanée de plusieurs parcelles, de la contiguïté des lots concernés,

- à défaut d'informations cadastrales, les transactions ont été localisées de manière aléatoire dans un rayon de $250 \mathrm{~m}$ autour du centroïde (barycentre) de la commune d'appartenance.

Les transactions recensées par la Safer sont analysées selon une segmentation par marché réalisée par la SCAFR (Société Centrale d'Aménagement Foncier Rural, organisme d'études et d'appui technique des Safer) selon un algorithme national qui retient comme principaux critères la nature cadastrale des parcelles, leur destination, leur taille et la nature des vendeurs et des acquéreurs. Trois segments sont retenus dans le présent article :

- le marché en vue de son artificialisation : les terres sont destinées à changer d'usage dans le cadre des règles d'urbanisme ;

- le marché des maisons à la campagne : il s'agit d'un sous-ensemble du marché foncier à usage résidentiel et de loisir concernant l'achat de biens libres bâtis de moins de 5 ha, acquis par des non-agriculteurs ;

- le marché des terres et prés : les terres achetées conservent leur usage de production primaire. 
L'ensemble des variables retenues dans les différentes estimations sont regroupées en blocs (cf. annexe 1). Des indicatrices temporelles (T) ont été systématiquement introduites dans les estimations, bien que le prix des transactions ait été déflaté, en utilisant l'indice de prix à la consommation (IPC) de l'Insee (base 100 en 2003) (5). L'ensemble des analyses traite donc de prix constants, en euros, de la transaction.

Les données Safer $(\mathrm{C})$ permettent de construire un ensemble de variables décrivant la nature de la transaction : surface du lot, présence et nature du bâti, caractéristiques des vendeurs et des acheteurs (6). Ces informations sont complétées par des variables provenant d'autres sources. Ainsi, les zonages en petites régions agricoles (PRA, source Insee), fournissent une information sur l'homogénéité culturale des communes permettant d'identifier une éventuelle logique spatiale d'origine agricole dans la formation des prix fonciers. Cavailhès et Wavresky (2003) retiennent ainsi cette variable comme indicatrice de la productivité agricole.

Par ailleurs, d'autres données à l'échelle communale (E), issues d'autres sources (Recensement Agricole, Corine Land Cover, Inao, IGN), apportent une information complémentaire sur les caractéristiques agricoles (part de la surface agricole et emprise de l'élevage mesurée par la superficie fourragère), environnementales (altitude des communes, part des surfaces humides et irriguées) ou urbaines (densité nette) de la commune dans laquelle a eu lieu la transaction.

Enfin, l'objectif de cette étude est, pour la première fois dans la littérature à notre connaissance, d'identifier la forme spatiale des gradients de rente foncière, c'est-à-dire la relation entre les prix fonciers et la distance au littoral, qui adopte des variations parfois éloignées de la traditionnelle décroissance exponentielle négative. Ainsi, des variables de distance (D) à la commune la plus fréquentée et au littoral complètent notre base. La distance au littoral a été calculée à l'aide d'un système d'information géographique (SIG) en prenant la distance euclidienne entre le point de localisation dans l'espace de la transaction et le trait de côte tel que défini par l'IGN en 2007. $\mathrm{La}$ " distance à la commune la plus fréquentée » est une variable issue de l'inventaire communal (Insee, 1998) et définie à partir des distances moyennes parcourues par les habitants pour accéder aux services et équipements.

La méthode des prix hédoniques constitue un cadre de modélisation pertinent de l'impact de littoralisation sur les valeurs foncières au sein des deux terrains d'études présentés plus haut. Cette méthode, largement développée en économie du logement à partir de l'article fondateur de Rosen (1974), permet d'estimer le prix implicite de la variation marginale d'une caractéristique d'un bien foncier ou immobilier (Sheppard, 1999). Le caractère marginal des modifications de caractéristiques est une condition particulièrement adaptée aux variables de distance (Anselin, 2008).

De manière à faciliter la comparaison des deux zones, la même spécification est retenue sur les deux sites. Après application de la transformation de Box-Cox (Box et Cox, 1964), le modèle prend la forme fonctionnelle log-linéaire suivante :

$$
\begin{aligned}
& \ln P_{i}=\alpha+\sum_{k} \eta_{k} T_{i}+\sum_{j} \beta_{j} C_{j, i}+\sum_{k} \delta_{k} E_{k, i} \\
& +\sum_{l} \gamma_{l} Z_{l, i}+\lambda_{m} D C_{m, i}+\phi D_{i}+\varepsilon_{i}
\end{aligned}
$$

où $\ln P_{i}$ est le logarithme du prix déflaté de la transaction relative au bien $i, T_{i}$ les variables discrètes temporelles $(k=2003$ à 2006 pour la Charente-Maritime et $k=2002$ à 2009 pour le Pays basque) (7) (8), $C_{j, i}$ les $j$ variables de contrôle concernant les caractéristiques des terres $i, E_{k, i}$ les $k$ variables de contrôle d'environnement issues des communes où est localisé le bien $i, Z_{l, i}$ les $l$ variables muettes qualifiant la zone dans laquelle se situe la transaction $i$ selon le zonage en PRA, $D_{i}$ la distance au littoral, $D C_{m, i}$ la distance à la commune la plus fréquentée $m$. Les $\varepsilon_{i}$ sont des résidus i.i.d. (indépendants et identiquement distribués) répondant aux propriétés habituelles. La surface des parcelles intervient au sein des caractéristiques $C$

5. L'IPC est un déflateur couramment utilisé. Le choix d'un indice relatif à l'immobilier n'apparait pas pertinent pour le traitement des marchés agricoles. Le choix alternatif du déflateur du PIB, lui aussi couramment mobilisé, présente peu de différences avec I'IPC et conduit à des estimations identiques, les différences résiduelles étant uniquement captées dans les indicatrices temporelles introduites systématiquement dans les spécifications. 6. Ces deux dernières caractéristiques influent généralement sur les prix à travers des pratiques pré - ou post - transaction qui échappent au marché, telles que le prêt de matériel, l'apport en travail, etc., lorsque l'acheteur et le vendeur sont tous deux des agriculteurs, pratiques concourant à une baisse, parfois sensible, du prix de la transaction. L'introduction de la profession des vendeurs et des acheteurs dans une régression hédonique s'en trouve légitimée.

7. Le processus de construction du modèle final nécessite un certain " tâtonnement " (inclusion/exclusion de variables), et notamment des variables croisées (indicatrices d'année, ou de la provenance et de la profession du vendeur et de l'acheteur, avec les variables de surface, de distance, etc.). Le modèle final ne les retient pas dans la mesure où elles n'étaient pas significatives.

8. L'influence des chocs exogènes (perturbation du marché de l'immobilier à partir de 2008) est prise en compte au moyen des indicatrices de l'année de transaction. 
sous forme logarithmique, conformément à une spécification habituelle liée à la non linéarité de la relation entre surface et prix des terrains et traduisant la constance de l'élasticité prix/surface sur chaque segment (Colwell et Munneke, 1997 ; Goffette-Nagot, 2009).

L'objet principal de cet article étant d'identifier l'existence et la forme des gradients de valeurs foncières littorales, rien ne justifie a priori une forme log-linéaire entre le prix et la distance au littoral. Un modèle de prix hédoniques semiparamétrique est donc spécifié, sur la base du modèle de Robinson (1988). Comme le suggèrent Parmeter et al. (2007), seules les variables d'intérêt spécifique, dans le cas présent la distance au littoral, intègrent le modèle sous forme non paramétrique, les autres demeurant sous forme linéaire.

Tableau 4

Estimations semi-paramétriques : significativité de $s(D)$

\begin{tabular}{|l|c|c|}
\hline \multicolumn{1}{|c|}{ Marché } & Côte basque & Littoral charentais \\
\hline Terres et prés & $10,25^{\star \star \star}$ & $5,97^{\star \star \star}$ \\
$\begin{array}{l}\text { Artificialisation } \\
\begin{array}{l}\text { Maisons à la } \\
\text { campagne }\end{array}\end{array}$ & $7,08^{\star \star \star}$ & $19,16^{\star \star \star}$ \\
\hline
\end{tabular}

Lecture : test de Fisher de significativité de la fonction non paramétrique $s(D)$. Les niveaux de risque des tests sont notés : $1 \%$ ,***, $5 \%{ }^{\prime * * \prime}$ et $10 \%$ "*'.

Champ : ensemble des transactions Safer échantillonnées par segment de marché et par terrain d'étude.

Source : traitement et calculs des auteurs d'après les données extraites des bases Safer.

$$
\begin{aligned}
& \ln P_{i}=\alpha+\sum_{k} \eta_{k} T_{i}+\sum_{j} \beta_{j} C_{j, i}+\sum_{k} \delta_{k} E_{k, i} \\
& +\sum_{l} \gamma_{l} Z_{l, i}+\lambda_{m} D C_{m, i}+s\left(D_{i}\right)+\varepsilon_{i}
\end{aligned}
$$

où la variable de distance au littoral $D$ influence le prix du terrain $i$ selon une fonction $s($.$) incon-$ nue. Cette spécification suggère l'utilisation de modèles semi-paramétriques additifs généralisés, dont l'estimation est détaillée dans l'encadré 2.

Les résultats relatifs à la partie non paramétrique (cf. tableau 4) de l'équation (2) indiquent l'existence d'une influence significative de la distance au littoral sur les prix sur chaque segment de marché étudié et sur chaque terrain d'étude. Les fonctions spline cubic $s(D)$ sont estimées sur chaque segment (cf. graphique II). Celles-ci suggèrent l'existence, sur la plupart des segments de marché, de relations linéaires fragmentées, jalonnées de changements de pentes (cf. encadré 3 ).

Un test complémentaire de non linéarité, le test de Davies (1987), permet de tester la significativité de tels changements de pente (cf. encadré 3). Sur le marché agricole de la côte basque, le test de Davies confirme la conclusion apportée plus haut à la lecture du graphique : le test ne rejette pas l'hypothèse d'un gradient de valeurs foncières décroissant et monotone (cf. tableau 5). C'est également le cas sur le

Encadré 2

\section{ESTIMATION SEMI-PARAMÉTRIQUE DES GRADIENTS DE VALEURS FONCIÈRES}

Les techniques d'estimation non paramétriques permettent de résoudre les difficultés de mauvaise spécification liées au choix des formes fonctionnelles, et permettent d'estimer les formes fonctionnelles les plus adéquates. Elles connaissent un succès croissant dans la littérature hédonique récente, notamment par l'application des régressions localement pondérées aux marchés immobiliers, depuis les travaux séminaux de Meese et Wallace (1991) et McMillen (1996). Les modèles additifs généralisés (GAM) présentent de nombreux avantages et apparaissent particulièrement adaptés à l'analyse hédonique spatiale (Geniaux et Napoleone, 2008). Ils constituent une extension du modèle de régression linéaire généralisée (GLM), permettant de spécifier la variable dépendante $\left(P_{j}\right)$ comme la somme d'une composante paramétrique $\left(\beta X_{i}\right)$ et d'une fonction lissée (smooth spline) de forme inconnue $s 0$ du régresseur $D$ (la distance au littoral dans cet article), soit :

$P_{i}=\beta X_{i}+s\left(D_{i}\right)+\varepsilon_{i}$
La fonction $s$ ( ) est estimée en recherchant une fonction $\hat{s}()$ deux fois dérivable minimisant la somme pénalisée des carrés des résidus, soit :

$\hat{s}=\operatorname{argmin} S S(\alpha)$

$S S(\alpha)=\sum_{i=1}^{n}\left\{P_{i}-s\left(D_{i}\right)\right\}^{2}+\alpha \int_{d_{\min }}^{d_{\max }}\left\{s^{\prime \prime}(D)\right\}^{2} d D$

où $\alpha$ désigne un paramètre de lissage commandant l'arbitrage entre qualité de l'ajustement et flexibilité de la fonction de lissage. Plusieurs algorithmes d'optimisation sont disponibles (Wood, 2008). La méthode d'optimisation utilisée dans cet article minimise le critère de validation croisée :

$\frac{n \sigma^{2}}{(n-D L)^{2}}$

où $n$ désigne le nombre d'observations, $\sigma^{2}$ la variance estimée des résidus et $D L$ le nombre équivalent de degrés de liberté du modèle. 
Graphique II

Forme non paramétrique de $s(D)$

A - Marché des terres destinées à l'artificialisation - Côte basque

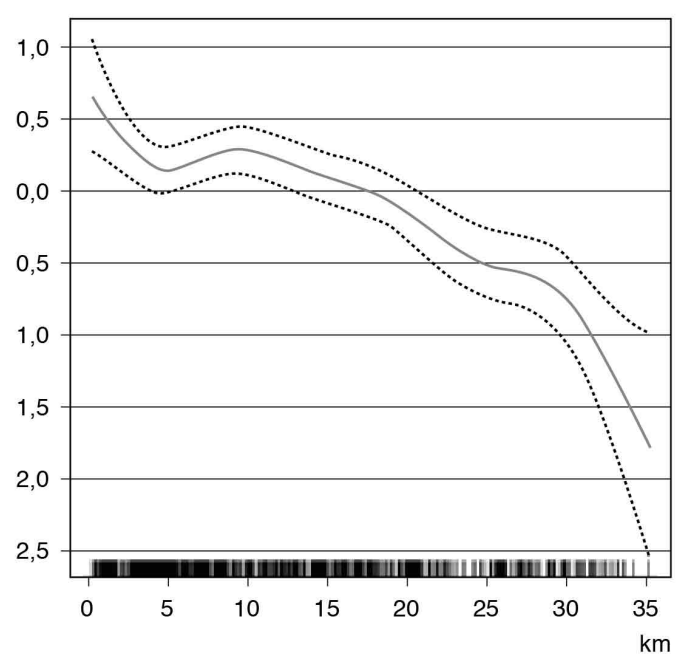

C - Marché des maisons à la campagne - Côte basque

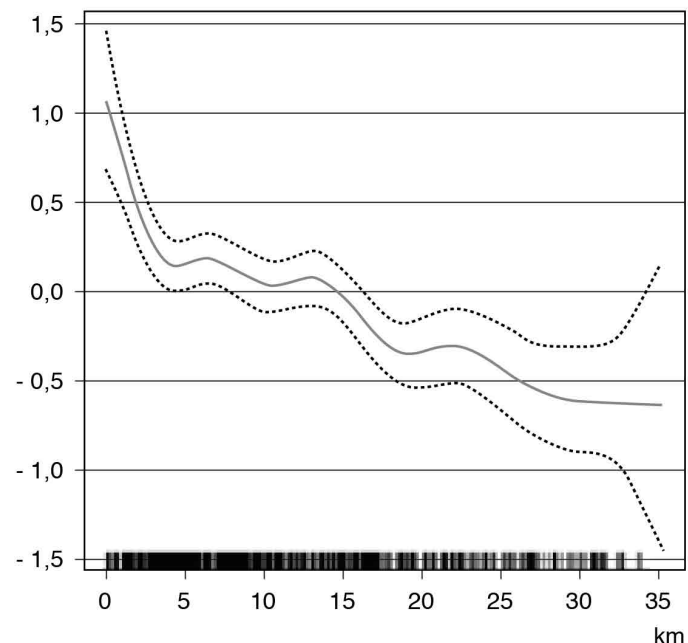

E - Marché des terres et prés - Côte basque

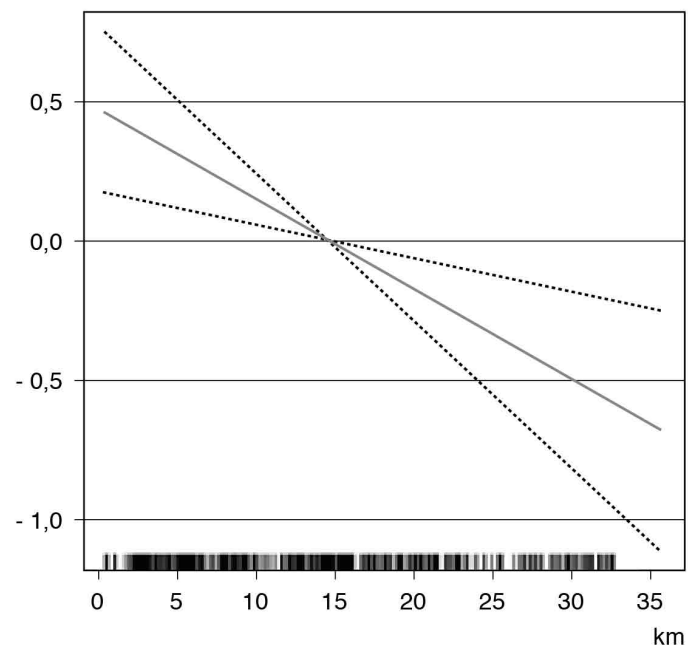

B - Marché des terres destinées à l'artificialisation - Littoral charentais

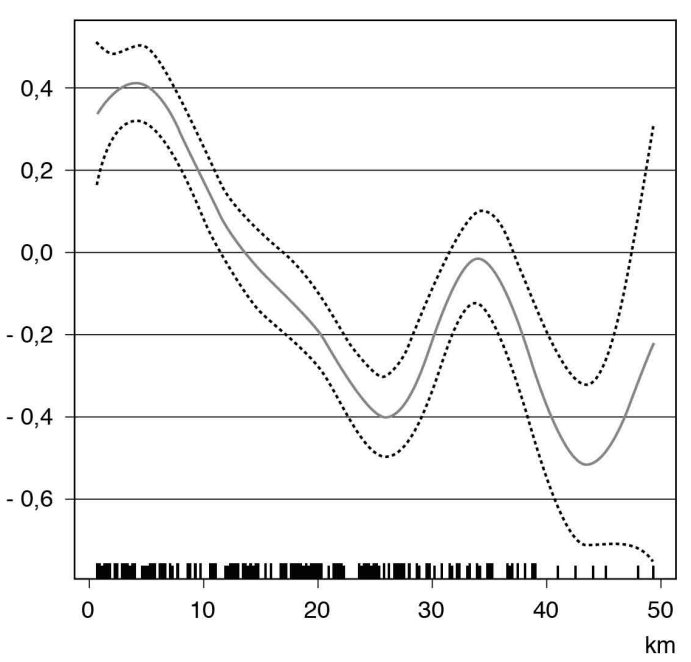

D - Marché des maisons à la campagne - Littoral charentais

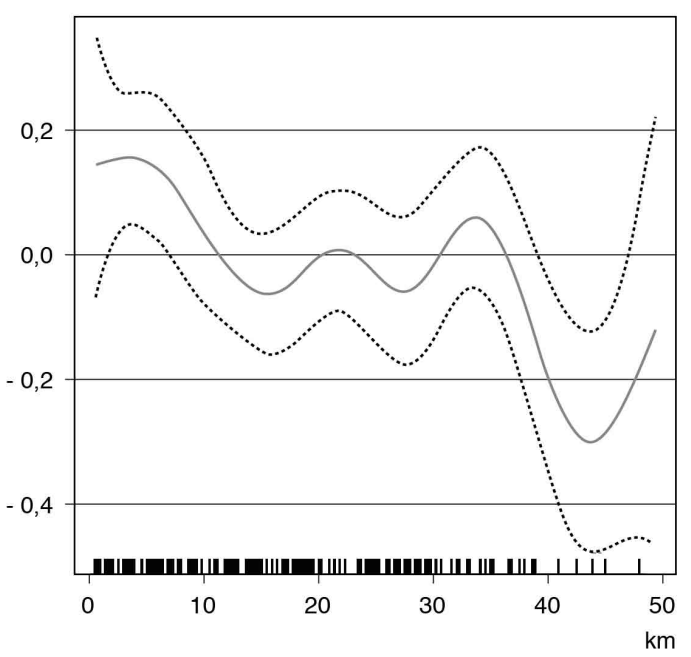

F - Marché des terres et prés - Littoral charentais

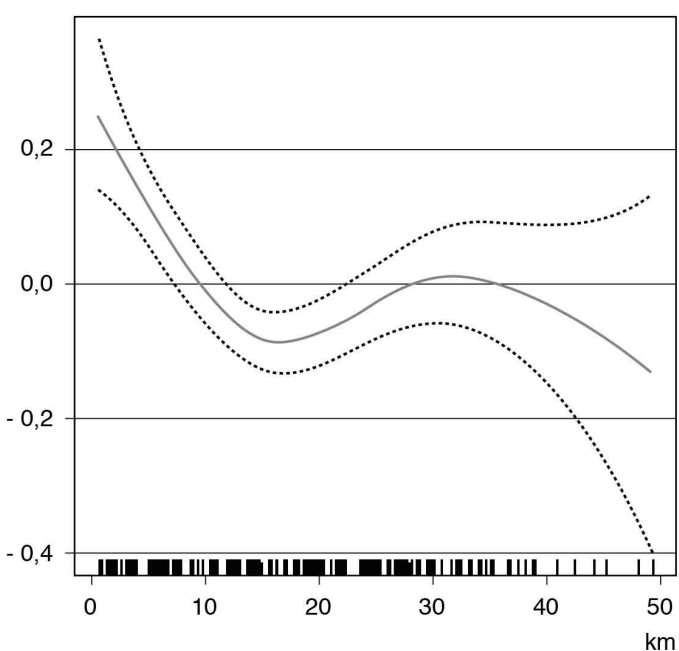

Lecture : le trait plein représente, sur chaque graphique, la relation non paramétrique estimée $s(D)$ entre le prix du sol (en ordonnées) et la distance au littoral (exprimée en $\mathrm{km}$ ), en abscisse. La distribution spatiale des observations est représentée par des traits verticaux le long de ce même axe. Les intervalles de confiance à $5 \%$ sont représentés en pointillés.

Champ : ensemble des transactions Safer échantillonnées par segment de marché et par terrain d'étude.

Source : traitement et calculs des auteurs d'après les données extraites des bases Safer. 
marché charentais des maisons où le test de Davies amène à conclure à une forme monotone du gradient (9).

La démarche retenue conduit à s'appuyer sur les estimations semi-paramétriques pour formuler des hypothèses quant à l'existence, au nombre et à la localisation présumée des changements de pente des gradients. L'analyse graphique est en effet réputée particulièrement importante dans les études empiriques de modèles semi- paramétriques (Bontemps et al., 2008). De plus, la méthode d'estimation retenue des relations linéaires fragmentées suppose que soit connue, au moins à titre d'hypothèse de départ, la localisation des changements de pentes estimés (cf. encadré 3).

9. Le graphe $D$ du graphique /l est cependant très heurté ce qui nous a incité à estimer une relation fragmentée. Celle-ci s'avérant non significative, nous avons choisi de maintenir une relation linéaire du prix à la distance au littoral.

Tableau 5

Distance au littoral : changements de pente estimés

\begin{tabular}{|c|c|c|c|c|}
\hline \multirow{2}{*}{ Marché } & \multirow{2}{*}{$\begin{array}{c}\text { Test de Davies } \\
p \text {-value }\end{array}$} & \multicolumn{3}{|c|}{ Distance au littoral (en $\mathrm{km}$ ) des changements de pente estimés } \\
\hline & & Break Point 1 & Break Point 2 & Break Point 3 \\
\hline \multicolumn{5}{|l|}{ Côte basque } \\
\hline Terres et prés & 0,1715 & - & - & - \\
\hline Artificialisation & 0,04154 & 0,95 & 12,29 & - \\
\hline Maisons à la campagne & 0,00026 & 1,78 & - & - \\
\hline \multicolumn{5}{|l|}{ Littoral charentais } \\
\hline Terres et prés & $6,175 e-05$ & 13,71 & 31,09 & - \\
\hline Artificialisation & $6,317 \mathrm{e}-05$ & 2,89 & 26,39 & 30,77 \\
\hline Maisons à la campagne & 0,9 & 14,64 & 24,03 & - \\
\hline
\end{tabular}

Lecture : test de significativité des changements de pente de la fonction $\mathrm{s}(\mathrm{D})$. Par exemple, sur le segment des Maisons à la campagne de la Côte basque, la fonction $\mathrm{s}(\mathrm{D})$ change de pente de façon significative (au seuil de $1 \%$ ) à 1,78 km du trait de côte.

Champ : ensemble des transactions Safer échantillonnées par segment de marché et par terrain d'étude.

Source : traitement et calculs des auteurs d'après les données extraites des bases Safer.

\section{Encadré 3}

\section{LES RÉGRESSIONS LINÉAIRES FRAGMENTÉES}

Les régressions linéaires fragmentées permettent une modélisation paramétrique des relations linéaires avec changements de pentes successifs d'une variable indépendante $D$ (la distance au littoral dans cet article).

Cette modélisation consiste à introduire un ensemble de variables muettes dans le modèle initial, pour chacun des points d'inflexion repérés :

$\ln P_{i}=\beta X_{i}+\alpha_{0} D_{i}+\sum_{k} \alpha_{k}\left(D_{i}-S_{k}\right) l_{K}+\varepsilon_{i}$

avec $I_{k}=1$ si $D_{i}>S_{k}, I_{k}=0$ sinon

où $I_{k}$ désignent les $k$ variables muettes correspondant aux $k$ points d'inflexion.

Muggeo (2003) a proposé une méthode itérative permettant de sélectionner les meilleurs seuils $S_{k}$, $k$ étant connu.

En s'appuyant sur la linéarisation suivante :

$\alpha_{0} D_{i}+\sum_{k} \alpha_{k}\left(D_{i}-S_{k}\right) l_{K}$

$=\alpha_{0} D_{i}+\sum_{k} \alpha_{k}\left(D_{i}-S_{k}^{(j)}\right) I_{K}-\left(S_{k}-S_{k}^{(j)}\right) I_{K}$
Il propose un algorithme itératif, qui, partant d'une valeur initiale $S_{k}^{(j)}$ de chaque seuil $k$ :

1) estime les paramètres $\hat{\alpha}_{k}$ et $\hat{\gamma}$ du modèle suivant par la méthode du maximum de vraisemblance :

$\ln P_{i}=\beta X_{i}+\alpha_{0} D_{i}+\sum_{k} \alpha_{k}\left(D_{i}-S_{k}^{(j)}\right) l_{K}-\gamma l_{K}+\varepsilon_{i}$

2) modifie le seuil $\mathrm{k}$ suivant la relation $s_{k}^{(j+1)}=\frac{\hat{\gamma}}{\hat{\beta}}+s_{k}^{(j)}$, jusqu'à convergence du processus, $\hat{\gamma}$ tendant vers 0 .

La fonction de vraisemblance n'étant pas nécessairement concave, il est nécessaire de tester plusieurs valeurs initiales des seuils $S_{k}$.

Le test proposé par Davies (1987) permet de tester, sur un ensemble $x$ de points successifs de l'intervalle $\left(d_{\text {min }}\right.$, $d_{\max }$ ) de la variable $d$, l'hypothèse nulle de nullité des paramètres $\alpha_{k}$ de changements de pente. II ne permet toutefois pas de choisir le nombre de changements de pentes à retenir dans la procédure d'estimation. 
Sur la base de l'analyse graphique, une procédure d'optimisation est utilisée pour localiser précisément les changements de pentes. Sur la base des résultats présentés au tableau 5 , les relations linéaires fragmentées sont estimées par la méthode des moindres carrés ordinaires (MCO) en spécifiant le modèle suivant :

$$
\begin{aligned}
& \ln P_{i}=\alpha+\sum_{k} \eta_{k} T_{i}+\sum_{j} \beta_{j} C_{j, i}+\sum_{k} \delta_{k} E_{k, i} \\
& \vdash \sum_{l} \gamma_{l} Z_{l, i}+\lambda_{m} D C_{m, i}+\rho_{1} D_{i} \\
& +\sum_{k} \rho_{k}\left(D_{i}-S_{k}\right) I_{k}+\varepsilon_{i}
\end{aligned}
$$

Le problème de l'endogénéité des variables explicatives se pose avec une acuité particulière dans les modèles hédoniques, certains attributs du bien étant co-déterminés avec le prix du bien sur le marché. De même, dans la mesure où l'unité d'observation est la transaction foncière, géolocalisée très précisément, des problèmes d'autocorrélation spatiale sont susceptibles de se poser. Le traitement économétrique de ces deux problèmes peut aujourd'hui se faire simultanément, au moyen de la procédure en trois étapes développée par Fingleton et Le Gallo (2008, cf. encadré 4).

Le choix d'une matrice de poids représentative des interdépendances spatiales est un problème délicat en économétrie appliquée. La pratique est de retenir la matrice de poids qui maximise l'indice de Moran (cf. annexe 2 pour les matrices de poids testées pour chaque site et pour chaque segment de marché), qui est l'indicateur global du degré d'autocorrélation entre unités spatiales (Le Gallo et al., 2003). La très faible différence des $I$ calculés (et de leur significativité) nous a conduits, par souci d'homogénéité dans la méthode d'estimation, à préférer systématiquement les matrices de distances-seuil, transformées en matrices de distance inverse au carré (cf. encadré 4).

L'ensemble des résultats des tests d'endogénéité et d'autocorrélation spatiale sont regroupés par segment de marché pour faciliter la lecture comparée des résultats (cf. annexes 3, 4 et 5).

\section{Encadré 4}

\section{ENDOGÉNÉITÉ ET AUTOCORRÉLATION SPATIALE : LA MÉTHODE FINGLETON-LE GALLO}

Le problème de l'endogénéité de la surface se pose de manière systématique dans les modèles de prix hédoniques, le choix simultané de la localisation et de la surface d'un terrain introduisant, du fait de la dépendance du prix à la localisation, une simultanéité potentielle entre prix et surface (Rapaport, 1997). Par ailleurs, les transactions foncières présentent habituellement un degré élevé d'interdépendance spatiale, nécessitant une modélisation adéquate.

La prise en compte simultanée de l'endogénéité et de l'autocorrélation spatiale est généralement limitée à l'introduction du décalage spatial de $1^{\text {er }}$ ordre de la variable expliquée, justifiant un recours aux doubles moindres carrés pour corriger l'endogénéité et l'introduction des décalages spatiaux de l'ensemble des variables exogènes comme instruments, selon la proposition de Kelejian et Prucha (1999a). Fingleton et Le Gallo (2008) ont étendu la procédure GS2SLS (Generalized Spatial Two-Stage Least Squares) de Kelejian et Prucha (1998), permettant la prise en compte simultanée d'un processus spatialement autorégressif de la variable expliquée et des erreurs, en autorisant également la prise en compte d'autres variables endogènes dans le modèle. La procédure proposée - FGS2SLS - (Feasible Generalized Spatial Two-Stage Least Squares) s'applique au modèle paramétrique (équation (3)) et comprend trois étapes :

1. Estimation d'un modèle autorégressif spatial par les doubles moindres carrés (IV-SAR), faisant appel aux variables instrumentales pour la variable endogène retardée spatialement (prix de la parcelle, noté W_LN_P) et pour le régresseur endogène (la surface, LN_SUR). En l'absence de variables exogènes susceptibles de constituer de bons instruments (les variables de profession des acheteurs et des vendeurs sont codées de manière trop fruste et comportent trop de valeurs manquantes), nous avons retenu ici, à l'instar de Fingleton et Le Gallo (2008), un quasi-instrument prenant les valeurs - 1, 0 ou 1 selon l'appartenance de l'observation au tercile inférieur, médian ou supérieur de la variable exogène.

2. Estimation par la méthode des moments généralisés de Kelejian et Prucha (1999) du paramètre $\lambda$ d'autorégression spatiale des erreurs à partir des résidus du modèle de première étape.

3. Ré-estimation par les 2SLS du modèle spécifié à l'étape 1 reformulé selon une transformation spatiale de Cochrane-Orcutt des variables à partir du paramètre estimé $\hat{\lambda}$. Une variable $X$ sera ainsi transformée de la manière suivante : $T_{-} X=[I-\hat{\lambda} W] X$ où I est une matrice identité et $\mathrm{W}$ une matrice de poids.

Pour chaque modèle (le IV-SAR de première étape, et le FGS2SLS de troisième étape), nous pratiquons plusieurs types de tests. Les tests d'endogénéité sont conduits à partir de statistiques robustes à l'hétéroscédasticité : le test $J$ de Hansen " de sur-identification » (égal à la statistique de Sargan sous condition 


\section{Résultats et discussion comparée autour des gradients de valeurs foncières}

Sur le segment des maisons à la campagne, la surface est exogène et les instruments valides. De plus, le test de nullité de l'indice de Moran de Anselin et Kelejian (1997) ne rejette pas l'absence d'autocorrélation spatiale résiduelle. Le modèle SAR estimé par les variables instrumentales apparaît donc adapté. Il conduit à estimer une forme spatialement autorégressive des valeurs foncières au Pays basque, le coefficient de la variable endogène décalée spatialement apparaissant significatif et de signe positif, traduisant l'existence d'effets de débordement locaux (i.e. le prix d'un terrain est influencé par le prix de ses voisins) qui induit une certaine segmentation spatiale du marché des maisons à la campagne au Pays basque (i.e. la segmentation du marché foncier se fait aussi sur un critère spatial). Cette structuration est absente sur la côte charentaise, dont le marché apparaît donc plus intégré.

Sur les segments de l'artificialisation et des terres agricoles, la présence d'autocorrélation spatiale ainsi que l'endogénéité de la surface justifient le recours au modèle FGS2SLS (cf. encadré 4). Les résultats obtenus conduisent systématiquement à un coefficient de la variable endogène décalée très faible et non significatif, traduisant l'absence de d'effets de débordement localisés sur ces marchés (10). Les tests de nullité de l'indice de Moran confirment dans la plupart des cas l'élimination de l'autocorrélation spatiale dans le résidu de troisième étape (11).

Précisons par ailleurs que les coefficients estimés pour les points d'inflexion («break points », indiqués par les variables $B P i$ ) n'indiquent pas la pente du gradient. En effet, la pente du $p^{e}$ fragment du gradient est calculée de la manière suivante :

pente $_{p}=\sum_{k=0}^{p} \alpha_{k}$

où $\alpha_{k}$ est le paramètre estimé correspondant au nœud $k$ de la distance au littoral (cf. tableau 6 pour les valeurs de ces pentes ainsi que les tests de Wald de nullité des pentes).

10. Ce résultat justifie l'estimation d'un modèle FGS2SLS ne prenant en compte que l'endogénéité de la surface et l'autocorrélation des erreurs, conduisant à des résultats similaires. Par souci d'homogénéité et de lisibilité des résultats nous maintenons la variable endogène décalée dans l'ensemble des spécifications présentées.

11. Le test de Moran conclut à l'absence d'autocorrélation spatiale résiduelle sur le marché agricole basque. La correction est néanmoins maintenue en raison du test de spécification d'Hausman qui, dans l'estimation de $\lambda$ par la méthode des moments généralisés, rejette à $5 \%$ l'hypothèse d'absence de dépendance spatiale dans les erreurs.

\section{Encadré 4 (suite)}

d'homoscédasticité) est pratiqué pour vérifier la validité des instruments retenus ; une statistique égale à celle d'Hausman sous condition d'homoscédascticité (C-test), permettant de statuer sur l'hypothèse d'exogénéité de la variable instrumentée (Hayashi, 2000). L'autocorrélation spatiale des résidus de modèles estimés par les doubles moindres carrés nécessite l'emploi d'un test spécifique adapté du / de Moran par Anselin et Kelejian (1997) (noté « I de Moran-VI »), les tests usuels de dépendance spatiale d'un modèle sans variables endogènes n'étant pas valides.

La matrice de poids spatiale, notée $W$, est une matrice carrée composée d'autant de lignes qu'il y a d'observations. Le terme générique $w_{i j}$ indique la façon dont les observations $i$ et $j$ sont connectées spatialement. Les éléments de la diagonale sont nuls par convention et la matrice est standardisée. Le choix de la matrice appropriée est une des difficultés méthodologiques majeures et les plus controversées en économétrie spatiale (Le Gallo, 2002). Un premier choix concerne la structure de voisinage retenue autour de chaque observation, qui peut être décrite de trois manières : matrices de contigüité reposant sur l'existence de frontières communes, matrices des $k$ plus proches voisins ou encore matrices de distance-seuil (distance euclidienne entre les centroïdes des entités). Le second choix concerne la pondération affectée à chacun des voisins retenus. $\mathrm{Si}$ la méthode usuelle consiste à affecter un poids égal à chacun d'entre eux, des contributions récentes, notamment en analyse hédonique spatiale (Baumont, 2009), retiennent plutôt une pondération inversement proportionnelle à la distance de chaque voisin, plus cohérente avec les hypothèses sous-jacentes des modèles hédoniques (variables omises à effet localisé ou mécanismes de diffusion locale des prix) (Anselin, 2008). Nous utilisons ici comme pondération l'inverse du carré de la distance, associée à une structure de voisinage de distance-seuil. La matrice obtenue se formalise de la manière suivante :

$$
\left\{\begin{array}{l}
w_{i j}^{*}(k)=0 \text { si } i=j, \forall k \\
w_{i j}^{*}(k)=1 / d_{i j}^{2} \text { si } d_{i j} \leq \bar{d} \quad \text { et } w_{i j}(k)=w_{i j}^{*}(k) \\
w_{i j}^{*}(k)=0 \text { si } d_{i j}>\bar{d}
\end{array}\right.
$$

où $w_{i j}(k)$ est l'élément de la matrice standardisée. Dans le cas d'une matrice de distance-seuil, $\bar{d}$ désigne la distance-seuil commune à toutes les observations, et définie ici de manière à ce que toutes les observations possèdent au moins un voisin. 
L'influence de la taille de la parcelle sur le prix du sol est conforme à celle attendue. Jouant identiquement sur tous les marchés, l'impact de la surface sur le prix est positif, mais l'élasticité du prix par rapport à la surface est sensiblement inférieure à l'unité, conformément aux résultats déjà obtenus dans la littérature (Colwell et Munneke, 1997 ; Cavailhès et Wavresky, 2003), à l'exception du marché des terres au Pays basque. Dans ce cas, l'élasticité est largement supérieure à l'unité : le prix implicite de l'hectare marginal est très fort sur ce marché, sur un terrain saturé où l'espace est très prisé. Une telle élasticité peut également s'interpréter comme un consentement à payer positif pour des parcelles remembrées, dans la mesure où les coûts marginaux d'exploitation sont plus faibles (Le Goffe, 2005). Bien qu'inférieure à l'unité, l'élasticité du prix par rapport à la surface sur le segment des terres agricoles en Charente-Maritime traduit de manière moins vive cette même logique : au-delà d'un certain seuil, le prix implicite de l'hectare marginal est plus faiblement valorisé sur le marché résidentiel, tandis que la superficie d'une exploitation agricole est un déterminant important de sa rentabilité. Enfin, les élasticités sont particulièrement faibles sur le segment des maisons à la campagne, la présence d'un bâtiment perturbant la relation surface-prix.

Les zonages en petites régions agricoles sont significativement négatifs sur les deux sites, tra- duisant les zones moins favorables à des cultures agricoles que la PRA de référence («Aunis » pour la Charente-Maritime et «Côte " pour le Pays basque, Cavailhès et Wavresky, 2003). En particulier, la culture dominante du maïs dans les PRA du Sud-Landes (Seignanx, Gosse, Marenne) semble provoquer un effet dépréciatif sur les valeurs foncières. Ces écarts de valorisation de PRA sont également marqués sur les marchés de l'artificialisation, traduisant la forte prime associée à des terres vendues à but d'artificialisation sur les PRA de référence. Ces derniers étant des espaces périurbains, on détecte ici l'influence de la composante urbaine sur les prix.

Sur les deux terrains d'étude, l'influence de la distance au littoral sur les prix fonciers est avérée quel que soit le segment de marché, même si la prime est d'une ampleur variable. Cela peut paraître étonnant dans la mesure où, on l'a souligné, la proximité au littoral n'a aucune raison objective d'influencer les prix fonciers sur le segment des terres purement agricoles. Cette influence proviendrait donc d'un mécanisme de transmission de la valeur entre les marchés fonciers et immobiliers. Ce résultat rentre en conformité avec ceux de Cavailhès et Wavresky (2003) qui, sur un terrain différent (le périurbain dijonnais), montrent que même le segment des terres agricoles est sujet aux perturbations provenant du marché immobilier.

Tableau 6

Tests de nullité des pentes pour les modèles retenus

\begin{tabular}{|c|c|c|c|}
\hline Segment de marché & Intervalle de distance $(\mathrm{km})$ & Pente & Statistique de Wald \\
\hline \multicolumn{4}{|l|}{ Artificialisation } \\
\hline \multirow{3}{*}{ Côte basque } & $0-1$ & $-2,9553$ & $15,06^{\star \star \star}$ \\
\hline & $1-12$ & 0,0133 & 0,51 \\
\hline & + de 12 & $-0,0566$ & $21,59^{\star \star \star}$ \\
\hline \multirow{4}{*}{ Littoral charentais } & $0-3$ & 0,0852 & 1,08 \\
\hline & $3-26$ & $-0,0388$ & $61,80^{\star \star \star}$ \\
\hline & $26-31$ & 0,0963 & $17,73^{\star \star \star}$ \\
\hline & $>31$ & $-0,0298$ & $10,15^{\star \star \star}$ \\
\hline \multicolumn{4}{|l|}{ Terres et prés } \\
\hline Côte basque & $0-35$ & $-0,0327$ & $7,58^{\star \star \star}$ \\
\hline \multirow{3}{*}{ Littoral charentais } & $0-14$ & $-0,0182$ & $6,11^{* *}$ \\
\hline & $14-31$ & 0,0097 & $6,70^{\star \star \star}$ \\
\hline & $>31$ & $-0,0093$ & 1,34 \\
\hline \multicolumn{4}{|l|}{ Maisons à la campagne } \\
\hline \multirow{2}{*}{ Côte basque } & $0-1,8$ & $-0,3250$ & $6,96^{\star \star \star}$ \\
\hline & $>1,8$ & $-0,0135$ & $3,36^{*}$ \\
\hline Littoral charentais & $0-49$ & $-0,0057$ & $7,92^{\star \star \star}$ \\
\hline
\end{tabular}

Lecture : la statistique de Wald est distribuée selon un F de Fisher pour les modèles estimés par les MCO et selon un $\chi^{2}(1)$ pour les modèles estimés par les doubles moindres carrés généralisés. Les niveaux de risque des tests sont notés : $1 \%$ "***', 5 \% "**' et $10 \%$ "**. Champ : ensemble des transactions Safer échantillonnées par segment de marché et par terrain d'étude.

Source : traitement et calculs des auteurs d'après les données extraites des bases Safer. 
De manière globale, le rythme de décroissance des prix avec l'éloignement au littoral est relativement comparable sur tous les segments en Charente-Maritime. Au contraire, les primes associées à la proximité au littoral sont extrêmement variables au Pays basque. On peut le relier, en première analyse, à des niveaux d'urbanisation et de pression foncière très différents. Ce constat demande néanmoins une analyse détaillée des gradients de prix foncier, à partir d'une comparaison entre le segment des terres agricoles et des terrains destinés à l'artificialisation.

C'est sur le segment de l'artificialisation que la prime liée à la proximité au littoral est la plus importante, un résultat conforme aux attendus théoriques. Le profil spatial de la prime est cependant très variable suivant le terrain d'étude.

Au Pays basque, elle est très élevée pour une étroite bande littorale de $1 \mathrm{~km}(-295 \% / \mathrm{km})$, un profil très semblable au segment des maisons à la campagne (- $33 \% / \mathrm{km}$ jusqu'à $1,8 \mathrm{~km})$. Un tel niveau de capitalisation s'explique par l'intensité des effets de rareté et d'aménité discutés plus haut sur la côte basque : la quasi-totalité de la bande littorale du Pays basque est d'ores et déjà artificialisée, et les terrains vacants sont peu nombreux ; d'autre part, la forte urbanisation de la bande d'exposition directe au littoral génère une forte valorisation des aménités paysagères. Le niveau très élevé de la prime résulte également de l'effet de levier sur les prix fonciers provoqué par le phénomène du compte à rebours.

Les prix fonciers deviennent insensibles à la distance au littoral entre 1 et $12 \mathrm{~km}$, ainsi que le confirme le test de Wald présenté au tableau 6 (12). Un tel résultat est contraire aux attendus théoriques de l'économie urbaine, fondés sur la compensation stricte des pertes d'accessibilité par une diminution du prix du sol. Ce raisonnement suppose cependant de prendre en compte les temps d'accès plutôt que la distance euclidienne. Or, les localisations situées à moins de $12 \mathrm{~km}$ du littoral correspondent à un temps de trajet inférieur à $30 \mathrm{~min}$, un seuil que l'on sait important dans les comportements de mobilité quotidienne. Ainsi, en deçà de ce seuil psychologique, les différentiels d'accessibilité ne sont pas capitalisés dans les prix fonciers : les localisations en deçà de $12 \mathrm{~km}$ du littoral sont dans une zone «d'accessibilité acceptable » au littoral et à ses activités économiques, et ne varient donc que peu en fonction de la distance phy- sique, d'autant que les aménités paysagères du front de mer ont disparu. Après $12 \mathrm{~km}$, le gradient reprend une forme plus attendue, décroissant à un taux de 5,6\%/ km.

Ces deux derniers résultats suggèrent que la structuration spatiale des prix fonciers se fait suivant une double logique : une logique d'aménité à proximité immédiate du littoral, une logique d'accessibilité au-delà, induisant un changement de pente brutal du gradient. $\mathrm{Ce}$ phénomène de décroissance brutale à proximité de l'aménité n'a jamais été observé avec une telle ampleur dans les gradients de prix fonciers périurbains, ce qui souligne le caractère exceptionnel des marchés fonciers littoraux. En revanche, les gradients résidentiels observés au-delà de la bande littorale $(-5,6 \%$ pour les terrains à bâtir, - 1,3\% pour les maisons à la campagne) sont conformes aux estimations présentées par Goffette-Nagot (2009) sur l'ensemble des espaces périurbains français $(-3,5 \%$ par $\mathrm{km})$, traduisant une logique d'accessibilité.

En Charente-Maritime, le gradient de prix sur le segment des terrains à bâtir adopte une forme sensiblement différente : d'abord croissant sur les trois premiers kilomètres (13), il adopte ensuite une décroissance à faible taux $(3,8 \%)$ jusqu'à $26 \mathrm{~km}$, puis il augmente à nouveau sur $5 \mathrm{~km}$, enfin il reprend sa décroissance à un taux comparable. Le rebond entre 26 et $31 \mathrm{~km}$ est probablement un artefact statistique, dû à l'effet inflationniste de la ville de Saintes, déjà noté dans la présentation des terrains d'étude. On notera en premier lieu la faiblesse du gradient par rapport à celui du Pays basque. Le double effet de marché n'est effectivement pas présent avec la même ampleur : du côté de l'offre, l'effet rareté joue beaucoup moins, car le littoral charentais est beaucoup moins artificialisé (cf. graphique I) ; du côté de la demande, les aménités littorales semblent moins valorisées, si l'on retient comme indicateurs d'attractivité le nombre de résidences secondaires ou l'activité touristique, tous deux beaucoup plus élevés sur la côte basque.

En second lieu, l'absence de lien entre la distance au littoral et le niveau des prix fonciers sur la bande des trois premiers kilomètres est

12. Cette fourchette de 1 à $12 \mathrm{~km}$ résulte de l'estimation paramétrique finale présentée au tableau 8 après sélection des points d'inflexion optimaux selon la procédure détaillée dans l'encadré 3 . Elle diffère légèrement de l'intervalle de 3 à $12 \mathrm{~km}$ visuellement suggéré par le graphique II.

13. La pente $n$ 'est toutefois pas significativement différente de 0 entre 0 et $3 \mathrm{~km}$ (cf. tableau 6). 
inattendue : si l'on se base sur les hypothèses théoriques comme sur les résultats obtenus pour le Pays basque, on devrait au contraire observer une accentuation de la pente à proximité immédiate du littoral. Ce résultat peut s'interpréter par les spécificités du territoire : le littoral charentais se caractérise par une forte proportion d'espaces humides (plus de $10 \%$ des surfaces sur la bande des cinq premiers kilomètres), ainsi que par une vulnérabilité importante aux tempêtes. Ainsi, les désaménités liées à une trop grande proximité au littoral semblent compenser les aménités.

Les plus-values de conversion anticipées sur le segment des terres agricoles sont théoriquement plus faibles, soit en raison de l'éloignement temporel de la perception de la rente, soit en raison de l'incertitude pesant sur la possibilité légale de la conversion découlant de l'inconstructibilité née de l'appartenance du terrain à un zonage particulier.

Sur ce segment, les gradients de prix ont sensiblement la même pente et la même forme sur les deux terrains d'étude. La décroissance continue du prix avec la distance au littoral peut s'expliquer par l'existence de plus-values de conversion anticipées intégrées dans les prix (14). L'influence positive de la densité résidentielle sur les prix de ce segment suggère la présence d'une « prime de croissance » dans les prix des terres agricoles. En outre, le prix de vente est plus faible lorsque l'acheteur est un établissement public : en effet, ces transactions échappent au marché et aux anticipations des agents privés, dans la mesure où le montant de la transaction est déterminé par une estimation des Domaines. Enfin, les prix sont insensibles à la temporalité : le segment des terres agricoles n'est pas influencé par la position dans le cycle immobilier, dans la mesure où le niveau des prix se nourrit des anticipations de plus-values, et non de leur montant actuel comme sur le segment des terrains à bâtir.

La proximité au littoral n'est capitalisée dans les prix fonciers agricoles que jusqu'à $13 \mathrm{~km}$ en Charente-Maritime : l'effet structurant du littoral a une portée moindre qu'au Pays basque. En outre, si la pente du gradient est relativement comparable à celle obtenue sur le segment de l'artificialisation en CharenteMaritime, elle est beaucoup plus faible au Pays basque, notamment sur la bande littorale. Cette différence de gradient entre les deux segments au Pays basque mérite une investigation supplémentaire.
Comme déjà souligné, l'existence d'un zonage rendant inconstructible certaines terres tend à réduire la composante urbaine des prix fonciers, par l'annulation des plus-values de conversion, sans pour autant la faire complètement disparaître en cas d'anticipation d'une révision prochaine du zonage ou d'un assouplissement de celui ci (règle de constructibilité limitée). Cette hypothèse se vérifie sur la côte basque, où $90 \%$ des terrains du segment agricole sont situés dans un zonage interdisant ou limitant la constructibilité (zones NC et ND) (15). Le caractère inconstructible des terres agricoles fournit ainsi une explication complémentaire à la faiblesse du gradient dans le Pays basque.

L'impact de la littoralisation sur les marchés fonciers dépend fortement des caractéristiques structurelles des deux sites étudiés. L'influence de la distance au littoral sur les prix fonciers est bien moindre en Charente-Maritime que sur la côte basque, notamment sur le segment de l'artificialisation. La présence de désaménités liées à des usages du sol particuliers sur le littoral charentais (zones humides), combinée au caractère beaucoup plus touristique de la côte basque, font que le littoral n'est pas valorisé de la même manière sur le marché foncier. Notre hypothèse est que le marché foncier charentais est marqué par son caractère agricole, tandis que celui du Pays basque est davantage orienté vers une logique résidentielle.

Ainsi, plusieurs variables de type agricole sont significatives en Charente-Maritime et peu au Pays basque. En Charente-Maritime, les terres agricoles qui se situent sur des communes plus irriguées sont plus chères, révélant le gain attendu de productivité lié à l'irrigation (Faux et Perry, 1999 ; Latinopoulos et al., 2004). Le nombre de labels enregistrés sur la commune (AOC, IGP, etc.) influence négativement le prix des terres à usage agricole mais positivement celles destinées à l'artificialisation. L'existence de labels peut être perçue par les acheteurs, dans le premier cas comme source de contraintes de production et donc d'accroissement des coûts, et dans le second cas plutôt comme un indice de qualité de l'environnement agricole du terrain à construire. Enfin, la part de surface fourragère de la commune joue positivement sur le mar-

14. Une explication alternative, proposée par Géniaux et Napoleone (2005), suggère de considérer les agriculteurs non pas seulement comme des producteurs, mais aussi comme des consommateurs d'aménités, à l'instar des autres résidents.

15. Cette statistique est obtenue en croisant la base de transactions Safer et les POS numérisés de la côte basque en vigueur au $1^{\text {er }}$ janvier 2006. 
ché de l'artificialisation, traduisant ici une sorte d'aménité paysagère liée à un paysage agricole alternatif aux grandes cultures plus habituelles. $\mathrm{Au}$ contraire, la part de surfaces agricoles a une influence négative sur le prix des terrains à bâtir de la côte basque, de même que la proportion de surfaces fourragères et l'altitude moyenne des communes.

Ces résultats contrastés soulignent que les aménités résidentielles ne sont pas de même type sur les deux territoires. Si les aménités littorales dominent au Pays basque, des aménités de type rurales sont davantage recherchées en Charente-Maritime, telles que le cadre de vie ou l'authenticité. Ainsi, le type de bâtiment d'une maison à la campagne joue sur les prix, là où il n'a aucune influence au Pays basque. D'autre part, la « distance à la commune la plus fréquentée » influence positivement le segment agricole en Charente-Maritime, traduisant une recherche d'aménités rurales (Bastian et al., 2002).

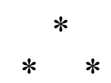

L'ambition de cet article était d'approfondir la relation entre la distance au littoral et les prix du sol. L'approche retenue est originale à deux niveaux : d'abord, par l'analyse et la comparaison de deux territoires distincts ; ensuite, par la mobilisation de techniques d'analyse semi-paramétriques complétées par la prise en compte de l'autocorrélation spatiale. Ces techniques nous ont permis de ne pas supposer, a priori, la forme fonctionnelle des gradients littoraux mais de la valider a posteriori. L'analyse précise de la forme spatiale du gradient de prix littoral, au cœur de cette contribution, montre l'existence d'une forte spécificité littorale sur nos deux espaces. Sur les marchés fonciers résidentiels de la côte basque, la forme particulière du gradient peut s'interpréter comme l'intégration d'une double logique de structuration de type aménités-accessibilité. En outre, sur nos deux espaces, les résultats valident l'existence de plus-values de conversion anticipées sur le marché des « terres et prés » qui dépendent du caractère constructible ou inconstructible de la parcelle. Pour autant, cette emprise de la littoralisation ne gomme pas les spécificités territoriales existantes. Ainsi, alors que sur le marché résidentiel basque la forme du gradient indique qu'une logique de rareté prévaut, liée notamment à une urbanisation plus avancée, sur la cote charentaise c'est une logique plus agricole, plus rurale qui prédomine mettant en lumière le rôle structurant des aménités territoriales.

Ce travail soulève des pistes d'approfondissement notamment concernant une prise en compte plus explicite de l'action publique (règlementations, zonage, etc.) dont le rôle structurant sur les dynamiques foncières est ici seulement évoqué en creux. Dans une perspective de plus long terme, les récents aléas climatiques qui ont touché la cote charentaise, nous interroge quant à la modification des comportements à venir, et l'effet de l'intégration de l'aversion au risque dans la structuration des prix fonciers.

\section{BIBLIOGRAPHIE}

Anselin L. (2008), "Spatial hedonics », in Mills T. C. and Patterson K., Palgrave handbook of econometrics, vol. 2, Palgrave MacMillan, Basingstoke.

Anselin L. et Florax R. (eds.) (1995), New Directions in Spatial Econometrics, SpringerVerlag.

Anselin L. and Kelejian H. H. (1997), « Testing for spatial error autocorrelation in the presence of endogenous regressors ", International Regional Science Review, $\operatorname{vol}^{\circ} 20$, pp. 153-182.

Ashton Morgan O. et Hamilton S. E. (2009), « Disentangling access and view amenities in access-restricted coastal residential communi- ties », Working Paper of the Appalachian State University, 09-10.

Bastian C.T., McLeod D.M., Germino M.J., Reiners W.A., and Blasko B.J. (2002), «Environmental Amenities and Agricultural Land Values : A Hedonic Model Using Geographic Information Systems Data ", Ecological Economics, vol. 40, pp. 337-349.

Baumont C. (2009), " Spatial effects of urban public policies on housing values ", Papers in Regional Science, vol. 88, n 2 , pp. 301-326.

Bin O., Crawford T. W., Kruse J. B., and Landry C. E. (2008), «Viewscapes and flood hazard : coastal housing market responses to ame- 
nities and risk », Land Economics, vol. 84, $\mathrm{n}^{\circ} 3$, pp. 434-448.

Bogart W. T. (2003), « Is zoning a substitute for, or a complement to factor taxes ? ", in Netzer D. (ed.), The Property tax, land use and land use regulation, Cheltenham, Edward Elgar.

Bontemps C., Simioni M., and Surry Y. (2008), «Semi parametric hedonic price models : assessing the effects of agricultural nonpoint source pollution ", Journal of applied econometrics, vol. 23, pp. 825-842.

Box G. et Cox D. (1964), « An analysis of transformation », Journal of the Royal Statistical Society, Series B (methodological), vol. 6, $\mathrm{n}^{\circ} 2$, pp. 211-252.

Brown G. M. et Pollakowski H. O. (1976), « Economic valuation of shoreline », Review of Economics and Statistics, vol. 59, n ${ }^{\circ} 3$, pp. 272278.

Capozza D. R. et Helsley R. W. (1989), « The fundamentals of land prices and urban growth », Journal of Urban Economics, vol. 26, pp. 295306.

Capozza D. R. et Li Y. (1994), « The intensity and timing of investment : the case of land $»$, American Economic Review, vol. 84, n 4, pp. 889-904.

Capozza D. R. and Helsley R. W. (1990), « The stochastic city », Journal of Urban Economics, vol. $28, \mathrm{n}^{\circ} 2$, pp. 187-203.

Cavailhès J. et Wavreski P. (2003), « Urban influences on periurban farmland prices », European Review of Agricultural Economics, vol. $30, \mathrm{n}^{\circ} 3$, pp. 333-357.

Cavailhès J., Hilal M., Wavresky P., Contesti G., et El Yousfi H. (2009), Marché foncier et périurbanisation, Rapport de recherche.

Cete Mediterranée (2007), Les évolutions des territoires littoraux, 1986-2006.

Cliff A. et Ord J.K. (1981), Spatial Processes : Models and Applications, Pion, London.

Cochrane D. et Orcutt G. H. (1949), « Application of least squares regression to relationships containing autocorrelated error terms », Journal of the American Statistical Association, vol. 44, pp. 32-61.
Colwell P. F. et Munneke H. J. (1997), « The Structure of Urban Land Prices », Journal of Urban Economics, vol. 41, pp. 321-336.

Comby J. (1995), « Enjeux fonciers sur le littoral », Colloque « Demain le rivage, un héritage à inventer », Assemblée Nationale, 14-15 novembre 1995.

Comby J. (1996), « Le compte à rebours de l'immeuble au terrain », Études Foncières, $n^{\circ} 73$, décembre.

Cunningham C. R. (2006), « House price uncertainty, timing of development, and vacant land prices. Evidence for real options in Seattle », Journal of Urban Economics, vol. 59, pp. 1-31.

Datar (2004), Construire ensemble un développement équilibré du littoral, La Documentation Française, Paris.

Davies R. B. (1987), « Hypothesis testing when a nuisance parameter is present only under the alternative », Biometrika, vol. 74, pp. 33-43.

Dawkins C.J., Nelson A. (2002), « Urban containment policies and housing prices : an international comparison with implications for future research $»$, Land Use Policy, vol. 19, n 1, pp. 1-12.

Diact (2007), « Bilan de la loi Littoral et des mesures en faveur du littoral », La documentation francaise, Paris.

Espace Rural (2006), « Le prix des terres en $2005 »$, Hors Série, mai.

Fareniaux B. et Verlhac E. (2008), « Le dysfonctionnement des marchés du logement en zone touristique », Rapport du Conseil Général des Ponts et Chaussées, $\mathrm{n}^{\circ}$ 004983-01, MEDAD.

Faux J. et Perry G.M. (1999), « Estimating irrigation water value using hedonic price analysis : a case study in Malheur county, Oregon », Land Economics, vol. 75, n 3, pp. 440-452.

Fingleton B. et Le Gallo J. (2008), « Estimating Spatial Models with Endogenous Variables, a Spatial Lag and Spatially Dependent Disturbances : Finite Sample Properties ", Papers in Regional Science, vol. 87, pp. 319-339.

Geniaux G. et Napoleone C. (2005), « Rente foncière et anticipations dans le périurbain ", Économie et Prévision, ${ }^{\circ}$ 68, pp. 77-93. 
Geniaux G. et Napoleone C. (2008), « Semiparametric tools for spatial hedonic models : an introduction to mixed geographically weighted regression and geoadditive models », in Baranzini, A., Ramirez, J., Schaerer, C. et Thalmann, P., Hedonic methods in housing Markets, New York, Springer.

Geoghegan J. (2002), « The value of open spaces in residential land use », Land Use Policy, $\mathrm{n}^{\circ} 19$, pp. 91-98.

Goffette-Nagot F. (2009), " Prix fonciers et demande de sol à usage résidentiel en France 1975-2000 », Revue Économique, vol. 60, pp. 853862.

Granelle J.-J. (1998), Économie Immobilière. Analyses et Applications, Paris, Economica.

Hayashi F. (2000), Econometrics, Princeton University Press.

Irwin E. G. et Bockstael N. E. (2004), « Land use externalities, open space preservation, and urban sprawl », Regional Science and Urban Economics, $\mathrm{n}^{\circ} 34$, pp. $705-725$.

Jaeger W.K. et Plantinga A.J. (2007), How Have Land-Use Regulations Affected Property Values in Oregon?, Oregon State University Extension Service, Special Report 1077.

Kelejian H. H. et Prucha I. R. (1998), "A Generalized Spatial Two-Stage Least Squares Procedure for Estimating a Spatial Autoregressive Model with Autoregressive Disturbances », Journal of Real Estate Finance and Economics, vol. $17, \mathrm{n}^{\circ} 1$, pp. $99-121$.

Kelejian H. H. et Prucha I. R. (1999), «A Generalized Moments Estimator for the Autoregressive Parameter in a Spatial Model "), International Economic Review, vol. 40, $\mathrm{n}^{\circ} 2$, pp. 509-533

Kerry Smith V. et Palmquist R. B. (1994), « Temporal substitution and the recreational value of coastal amenities ", Review of Economics and Statistics, vol. 76, n 1, pp. 119-126.

Latinopoulos P., Tziakas V., and Mallios Z. (2004), "Valuation of Irrigation Water by the Hedonic Price Method : A Case Study in Chalkidiki, Greece », Water, Air, \& Soil Pollution : Focus, vol. 4, n 4, pp. 253-262.

Le Gallo J., (2002), « Économétrie spatiale : l'autocorrélation spatiale dans les modèles de régression linéaire », Économie et Prévision, vol. $155, \mathrm{n}^{\circ} 4$, pp. $139-158$.

Le Goffe P. et Salanié J. (2005), « Le droit d'épandage a-t-il un prix ? Mesure sur le marché foncier », Cahiers d'Économie et Sociologie Rurales, $\mathrm{n}^{\circ} 77$, pp. 36-63.

Lecat G. (2006), Analyse économique de la planification urbaine, Thèse ès Sciences Économiques, Université de Bourgogne.

McMillen D. P. (1996), " One hundred fifty years of land values in Chicago : a nonparametric approach », Journal of Urban Economics, vol. 40, pp. 100-124.

Meese R. et Wallace N. (1991), « Nonparametric estimation of dynamic hedonic price models and the construction of residential housing price indices », American Real Estate and Urban Economic Association Journal, vol. 19, pp. 308-332.

Mori H. (1998), « Land Conversion at the Urban Fringe : A Comparative Study of Japan, Britain and the Netherlands ", Urban Studies, vol. 35, pp. 1541-1558.

Muggeo V. (2003), « Estimating regression models with unknown break-points », Statistics in Medicine, vol. 22, pp. 3055-3071.

Parmeter C. F., Henderson D. J., and Kumbhakar S. C. (2007), « Nonparametric estimation of a hedonic price function », Journal of Applied Econometrics, vol. 22, pp. 695-699.

Parsons G. R. et Noailly J. (2004), « A value capture tax for financing beach nourishment projects : an application to Delaware's ocean beaches ", Ocean and Coastal Management, $\mathrm{n}^{\circ} 47$, pp. 49-61.

Plantinga A. J. et Miller D. J. (2001), " Agricultural land values and the value of rights to future land development », Land Economics, vol. $77, \mathrm{n}^{\circ} 1$, pp. 56-67.

Plantinga A. J., Lubowski R. N., and Stavins R. N. (2002), « The effects of potential land development on agricultural land prices », Journal of Urban Economics, $\mathrm{n}^{\circ}$ 52, pp. 561-581.

Polomé P., Marzetti S., and van der Veen A. (2005), «Economic and social demands for coastal protection », Coastal Engineering, $\mathrm{n}^{\circ} 52$, pp. 819-840. 
Pompe J. J. (2008), « The effect of a gated community on property and beach amenity valuation $"$, Land Economics, vol. 84, n 3, pp. 423-433.

Rapaport C. (1997), « Housing demand and community choice : an empirical analysis », Journal of Urban Economics, vol. 42, pp. 243-260.

Robinson P. M. (1988), « Root-N-consistent semiparametric regression », Econometrica, vol. 56, pp. 931-954.

Rosen S. (1974), « Hedonic prices and implicit markets : product differentiation in perfect competition », Journal of Political Economy, vol. 82, pp. 34-55.

Segerson K., Plantinga A. J., and Irwin E. G. (2006), « Theoretical background », in Bell K. P.,
Boyle K. J., Rubin J. (eds), Economics of rural land-use change, Ashgate, Aldershot, pp. 79-112.

Sheppard S. (1999), « Hedonic analysis of housing markets », in C. Cheshire et E. S. Mills (eds), Handbook of regional and urban economics, Elsevier, vol. 3, chap. 41, pp. 1595-1635

Travers M., Nassiri A., Appere G., et Bonnieux F. (2008), " Évaluation des bénéfices environnementaux par la méthode des prix hédonistes : une application au cas du littoral », Économie et Prévision, $\mathrm{n}^{\circ} 185$, pp. 47-62.

Wood S.N. (2008), « Fast stable direct fitting and smoothness selection for generalized additive models », Journal of the Royal Statistical Society, vol. $70, \mathrm{n}^{\circ} 3$, pp. 495-518. 
Liste des variables utilisées dans les modèles

\begin{tabular}{|c|c|c|c|c|c|c|c|c|}
\hline \multirow[b]{2}{*}{ Variables } & \multirow[b]{2}{*}{ Définition de la variable } & \multirow[b]{2}{*}{ Intitulé de la variable } & \multirow[b]{2}{*}{ Unité } & \multirow[b]{2}{*}{ Source } & \multicolumn{2}{|c|}{ Côte basque } & \multicolumn{2}{|c|}{ Littoral charentais } \\
\hline & & & & & Moyenne & $\begin{array}{l}\text { Écart- } \\
\text { type }\end{array}$ & Moyenne & $\begin{array}{l}\text { Écart- } \\
\text { type }\end{array}$ \\
\hline \multirow[t]{8}{*}{$\mathrm{T}$} & \multirow[t]{8}{*}{ Variables muettes de l'année de transaction } & X2002 & $1=$ oui $/ 0=$ non & \multirow[t]{19}{*}{ Safer } & 0,12 & - & - & - \\
\hline & & X2003 & $1=$ oui $/ 0=$ non & & 0,12 & - & 0,22 & - \\
\hline & & X2004 & $1=$ oui $/ 0=$ non & & 0,13 & - & 0,25 & - \\
\hline & & X2005 & $1=$ oui $/ 0=$ non & & 0,13 & - & 0,26 & - \\
\hline & & X2006 & $1=$ oui $/ 0=$ non & & 0,13 & - & 0,26 & - \\
\hline & & X2007 & $1=$ oui $/ 0=$ non & & 0,15 & - & - & - \\
\hline & & X2008 & $1=$ oui $/ 0=$ non & & 0,11 & - & - & - \\
\hline & & X2009 & $1=$ oui $/ 0=$ non & & 0,10 & - & - & - \\
\hline \multirow[t]{11}{*}{$\mathrm{C}$} & Surface de la parcelle vendue & SURF & Hectare & & 1,38 & 2,31 & 1,64 & 3,41 \\
\hline & $\begin{array}{l}\text { Variable muette de présence d'un bâti sur la } \\
\text { parcelle }\end{array}$ & BATI & $1=$ oui $/ 0$ = non & & 0,35 & - & 0,25 & - \\
\hline & \multirow{3}{*}{$\begin{array}{l}\text { Variables muettes sur le type de bâti présent } \\
\text { sur la parcelle : bâtiment d'exploitation, } \\
\text { d'habitation ou mixte }\end{array}$} & BAT_EXP & $1=$ oui $/ 0=$ non & & 0,01 & - & 0,03 & - \\
\hline & & BAT_HAB & $1=$ oui $/ 0=$ non & & 0,30 & - & 0,85 & - \\
\hline & & BAT_MIXTE & $1=$ oui $/ 0=$ non & & 0,04 & - & 0,07 & - \\
\hline & \multirow{2}{*}{$\begin{array}{l}\text { Variables muettes caractérisant l'origine } \\
\text { régionale de l'acheteur ou du vendeur } \\
\text { (Poitou-Charentes/Aquitaine) }\end{array}$} & V_AQUI/CHARENTE & $1=$ oui $/ 0=$ non & & 0,87 & - & 0,88 & - \\
\hline & & A_AQUI/CHARENTE & $1=$ oui $/ 0=$ non & & 0,83 & - & 0,87 & - \\
\hline & \multirow{4}{*}{$\begin{array}{l}\text { Variables muettes caractérisant la qualité } \\
\text { d'établissement public de l'acheteur ou du } \\
\text { vendeur } \\
\text { Variables muettes caractérisant l'activité de } \\
\text { l'acheteur ou du vendeur }\end{array}$} & V_ETABPUB & $1=$ oui $/ 0=$ non & & 0,04 & - & 0,03 & - \\
\hline & & A_ETABPUB & $1=$ oui $/ 0=$ non & & 0,02 & - & 0,07 & - \\
\hline & & V_PROFAGRI & $1=$ oui $/ 0=$ non & & 0,13 & - & 0,14 & - \\
\hline & & A_PROFAGR & $1=$ oui $/ 0=$ non & & 0,13 & - & 0,37 & - \\
\hline \multirow[t]{12}{*}{ Z } & \multirow{12}{*}{$\begin{array}{l}\text { Variables muettes qualifiant la zone dans } \\
\text { laquelle se situe la transaction selon le } \\
\text { zonage en PRA }\end{array}$} & PRA_AUNIS & $1=$ oui $/ 0=$ non & \multirow[t]{12}{*}{ Insee } & & & 0,26 & - \\
\hline & & PRA_ROCHEF & $1=$ oui $/ 0=$ non & & & & 0,13 & - \\
\hline & & PRA_POITEVIN & $1=$ oui $/ 0=$ non & & & & 0,05 & - \\
\hline & & PRA_SAINTAG & $1=$ oui $/ 0=$ non & & & & 0,15 & - \\
\hline & & PRA_SAINTVIT & $1=$ oui $/ 0=$ non & & & & 0,41 & - \\
\hline & & PRA_COTE & $1=$ oui $/ 0=$ non & & 0,17 & - & & \\
\hline & & PRA_MARENN & $1=$ oui $/ 0=$ non & & 0,09 & - & & \\
\hline & & PRA_GOSSE & $1=$ oui $/ 0=$ non & & 0,11 & - & & \\
\hline & & PRA_SEIGNA & $1=$ oui $/ 0=$ non & & 0,07 & - & & \\
\hline & & PRA_COTEAUX & $1=$ oui $/ 0=$ non & & 0,35 & - & & \\
\hline & & PRA_VALADO & $1=$ oui $/ 0=$ non & & 0,19 & - & & \\
\hline & & PRA_MONTAG & $1=$ oui $/ 0=$ non & & 0,02 & - & & \\
\hline \multirow[t]{7}{*}{$E$} & $\begin{array}{l}\text { Part de Surface Agricole Utile de la } \\
\text { commune de la transaction }\end{array}$ & SAU & $\begin{array}{l}\text { \% surface } \\
\text { commune }\end{array}$ & RA 2000 & 0,43 & 0,20 & 0,67 & 0,16 \\
\hline & $\begin{array}{l}\text { Part de superficie fourragère principale dans } \\
\text { la SAU de la commune }\end{array}$ & PSAU_FOURR & $\begin{array}{l}\text { \% surface } \\
\text { commune }\end{array}$ & & 0,67 & 0,31 & 0,20 & 0,17 \\
\hline & $\begin{array}{l}\text { Nombre total de labels de la commune de } \\
\text { transaction }\end{array}$ & NB_LABELS & Nombre & Inao & 12,85 & - & 8 & - \\
\hline & $\begin{array}{l}\text { Part de surface irriguée dans la SAU de la } \\
\text { commune de la transaction }\end{array}$ & P_IRRIG & $\begin{array}{c}\% \text { SAU } \\
\text { communale }\end{array}$ & RA 2000 & - & - & 17,41 & 14,22 \\
\hline & Altitude de la commune de la transaction & ALTMEAN & mètres & IGN-MNT 2008 & 83,34 & 77,20 & - & - \\
\hline & $\begin{array}{l}\text { Part de zones humides dans la surface de la } \\
\text { commune }\end{array}$ & P_ZH & $\begin{array}{l}\% \text { surface } \\
\text { commune }\end{array}$ & CLC 2006 & 0,00 & 0,01 & 2,06 & 7,40 \\
\hline & $\begin{array}{l}\text { Indicateur de pression sur les espaces } \\
\text { habitables en } 2006\end{array}$ & DENSNETTE & Habitants $/ \mathrm{km}^{2}$ & & 250,15 & 362,93 & 145,34 & 249,89 \\
\hline
\end{tabular}


Liste des variables utilisées dans les modèles (suite)

\begin{tabular}{|c|c|c|c|c|c|c|c|c|}
\hline \multirow[b]{2}{*}{ Variables } & \multirow[b]{2}{*}{ Définition de la variable } & \multirow[b]{2}{*}{ Intitulé de la variable } & \multirow[b]{2}{*}{ Unité } & \multirow[b]{2}{*}{ Source } & \multicolumn{2}{|c|}{ Côte basque } & \multicolumn{2}{|c|}{ Littoral charentais } \\
\hline & & & & & Moyenne & $\begin{array}{l}\text { Écart- } \\
\text { type }\end{array}$ & Moyenne & $\begin{array}{l}\text { Écart- } \\
\text { type }\end{array}$ \\
\hline $\mathrm{D}$ & $\begin{array}{l}\text { Distance en kilomètres à la commune la plus } \\
\text { fréquentée }\end{array}$ & D_CFREQ & $\mathrm{km}$ & $\begin{array}{c}\text { Inventaire } \\
\text { communal } \\
1998\end{array}$ & 12,18 & 7,77 & 10,58 & 6,86 \\
\hline & Distance en kilomètres au littoral & DLIT & $\mathrm{km}$ & SIG & 13,32 & 8,76 & 16,75 & 11,49 \\
\hline
\end{tabular}

Lecture : les variables ont été regroupées en sous-groupes, notées par une lettre en majuscule par souci de simplification dans l'écriture des équations : $T$ = variables temporelles ; $C=$ caractéristiques du bien, de l'acheteur et du vendeur ; $Z=$ zonage en PRA ; $E=$ caractéristiques de l'environnement du bien ; $D=$ variables de distance. La variable DLIT peut éventuellement être notée BPi (break point) dans certains modèles. En effet, en présence de break points (dont la significativité est testée dans le tableau 5, DLIT est scindée en plusieurs variables, chacune correspondant à l'intervalle dont la borne est un break point. Source : auteurs. 
ANNEXE 2

Analyse de l'autocorrélation spatiale : test de nullité du I de Moran pour différentes matrices de poids

\begin{tabular}{|c|c|c|c|c|c|c|c|c|c|c|c|c|}
\hline \multirow{3}{*}{$\begin{array}{l}\text { Critère de } \\
\text { distance }\end{array}$} & \multicolumn{4}{|c|}{ Artificialisation } & \multicolumn{4}{|c|}{ Maisons à la campagne. } & \multicolumn{4}{|c|}{ Terres et prés } \\
\hline & \multicolumn{2}{|c|}{ Côte basque } & \multicolumn{2}{|c|}{ Littoral charentais } & \multicolumn{2}{|c|}{ Côte basque } & \multicolumn{2}{|c|}{ Littoral charentais } & \multicolumn{2}{|c|}{ Côte basque } & \multicolumn{2}{|c|}{ Littoral charentais } \\
\hline & I & $p$-value & 1 & $p$-value & 1 & $p$-value & 1 & p-value & I & $p$-value & 1 & $p$-value \\
\hline 4 & 0,376 & $<2,2 \mathrm{e}^{-16}$ & 0,177 & $<2,2 \mathrm{e}^{-16}$ & 0,36 & $<2,2 \mathrm{e}^{-16}$ & 0,005 & 0,79 & 0,284 & $<2,2 \mathrm{e}^{-16}$ & 0,064 & $8,23 e^{-05}$ \\
\hline 8 & 0,371 & $<2,2 \mathrm{e}^{-16}$ & 0,176 & $<2,2 \mathrm{e}^{-16}$ & 0,367 & $<2,2 \mathrm{e}^{-16}$ & 0,009 & 0,64 & 0,288 & $<2,2 \mathrm{e}^{-16}$ & 0,067 & $4,41 \mathrm{e}^{-06}$ \\
\hline 10 & 0,367 & $<2,2 \mathrm{e}^{-16}$ & 0,174 & $<2,2 \mathrm{e}^{-16}$ & 0,364 & $<2,2 \mathrm{e}^{-16}$ & 0,010 & 0,61 & 0,287 & $<2,2 \mathrm{e}^{-16}$ & 0,068 & $1,66 e^{-06}$ \\
\hline 20 & 0,362 & $<2,2 \mathrm{e}^{-16}$ & 0,175 & $<2,2 \mathrm{e}^{-16}$ & 0,363 & $<2,2 \mathrm{e}^{-16}$ & 0,011 & 0,55 & 0,281 & $<2,2 \mathrm{e}^{-16}$ & 0,069 & $3,83 e^{-07}$ \\
\hline 50 & 0,357 & $<2,2 \mathrm{e}^{-16}$ & 0,174 & $<2,2 \mathrm{e}^{-16}$ & 0,358 & $<2,2 \mathrm{e}^{-16}$ & 0,012 & 0,53 & 0,27 & $<2,2 \mathrm{e}^{-16}$ & 0,068 & $2,96 \mathrm{e}^{-07}$ \\
\hline d-seuil & 0,378 & $<2,2 \mathrm{e}^{-16}$ & 0,175 & $<2,2 \mathrm{e}^{-16}$ & 0,368 & $<2,2 \mathrm{e}^{-16}$ & 0,012 & 0,54 & 0,279 & $<2,2 \mathrm{e}^{-16}$ & 0,068 & $3,20 e^{-07}$ \\
\hline Valeurd-seuil & \multicolumn{2}{|c|}{$2,944 \mathrm{~km}$} & \multicolumn{2}{|c|}{$4,198 \mathrm{~km}$} & \multicolumn{2}{|c|}{$4,218 \mathrm{~km}$} & \multicolumn{2}{|c|}{$4,147 \mathrm{~km}$} & \multicolumn{2}{|c|}{$5,175 \mathrm{~km}$} & \multicolumn{2}{|c|}{$3,124 \mathrm{~km}$} \\
\hline
\end{tabular}

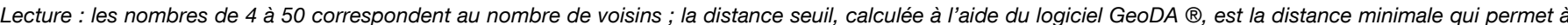
chaque observation d'avoir au moins un voisin.

Champ : ensemble des transactions Safer échantillonnées par segment de marché et par terrain d'étude.

Source : traitement et calculs des auteurs d'après les données extraites des bases Safer. 
ANNEXE 3

Résultats des estimations sur le marché des maisons à la campagne

\begin{tabular}{|c|c|c|c|c|c|c|c|c|}
\hline & & Côte & asque & & & Littoral & narentais & \\
\hline & & & & & & & & \\
\hline Constante & $13,31^{\star \star \star}$ & $(26,46)$ & $9,25^{\star \star \star}$ & $(6,54)$ & $10,53^{\star \star \star}$ & $(19,01)$ & $9,90^{\star \star \star}$ & $(9,59)$ \\
\hline WLN_P & - & - & $0,33^{\star \star \star}$ & $(3,76)$ & - & - & 0,05 & $(0,75)$ \\
\hline X2002 & $-0,38^{\star \star \star}$ & $(-3,01)$ & $-0,34^{\star \star \star}$ & $(-2,91)$ & - & - & - & - \\
\hline X2003 & $-0,21^{*}$ & $(-1,67)$ & $-0,20^{*}$ & $(-1,7)$ & Ref & Ref & Ref & Ref \\
\hline X2004 & $-0,23^{*}$ & $(-1,79)$ & $-0,19^{\star}$ & $(-1,66)$ & $0,20^{\star \star \star}$ & $(3,37)$ & $0,20^{\star \star}$ & $(3,43)$ \\
\hline X2005 & 0,11 & $(0,91)$ & 0,14 & $(1,18)$ & $0,29^{\star \star \star}$ & $(5,05)$ & $0,29^{\star \star \star}$ & $(5,04)$ \\
\hline X2006 & 0,17 & $(1,37)$ & $0,21^{*}$ & $(1,83)$ & $0,33^{\star \star \star}$ & $(5,96)$ & $0,35^{\star \star \star}$ & $(5,70)$ \\
\hline X2007 & 0,13 & $(1,09)$ & 0,17 & $(1,51)$ & - & - & - & - \\
\hline X2008 & 0,11 & $(0,83)$ & 0,13 & $(1,06)$ & - & - & - & - \\
\hline LN_SUR & $0,28^{\star \star \star}$ & $(8,7)$ & $0,26^{\star \star \star}$ & $(8,22)$ & $0,24^{\star \star \star}$ & $(13,06)$ & $0,24^{\star \star \star}$ & $(11,32)$ \\
\hline BAT_HAB & 0,24 & $(1,14)$ & 0,32 & $(1,64)$ & $1,96^{\star \star \star}$ & $(17,96)$ & $1,96^{\star \star \star}$ & (9) \\
\hline BAT_MIXTE & 0,34 & $(1,55)$ & $0,42^{\star \star}$ & $(2,02)$ & $1,77^{\star \star \star}$ & $(13,51)$ & $1,77^{\star \star \star}$ & $(7,74)$ \\
\hline A_AQUI / CHARENTE & $-0,35^{\star \star *}$ & $(-5,94)$ & $-0,33^{\star \star \star}$ & $(-5,86)$ & $-0,31^{\star \star \star}$ & $(-7,31)$ & $-0,31^{\star \star \star}$ & $(-7,82)$ \\
\hline V_AQUI / CHARENTE & $-0,01$ & $(-0,1)$ & 0,01 & $(0,12)$ & $0,17^{\star \star \star}$ & $(2,89)$ & $0,16^{\star \star \star}$ & $(2,84)$ \\
\hline V_PROFAGRI & $-0,42^{\star \star \star}$ & $(-3,48)$ & $-0,36^{\star \star \star}$ & $(-3,28)$ & $-0,31^{\star \star *}$ & $(-3,61)$ & $-0,31^{\star \star *}$ & $(-2,87)$ \\
\hline A_PROFAGR & - & - & - & - & $-0,96^{\star \star}$ & $(-2,36)$ & $-0,96^{\star \star}$ & $(-1,47)$ \\
\hline V_ETABPUB & - & - & - & - & 0,38 & $(0,79)$ & 0,38 & $(0,39)$ \\
\hline A_ETABPUB & - & - & - & - & $-0,17$ & $(-0,62)$ & $-0,18$ & $(-0,45)$ \\
\hline PRA_MARENN/ROCHEF & $-0,61^{\star \star \star}$ & $(-3,35)$ & $-0,33^{*}$ & $(-1,86)$ & $-0,30^{\star \star}$ & $(-2,55)$ & $-0,28^{\star \star}$ & $(-2,25)$ \\
\hline PRA_GOSSE/POITEVIN & $-0,53^{\star \star \star}$ & $(-3,27)$ & $-0,30^{*}$ & $(-1,93)$ & $-0,33^{\star \star \star}$ & $(-2,887)$ & $-0,31^{\star \star \star *}$ & $(-2,79)$ \\
\hline PRA_SEIGNA/SAINTAGR & $-0,50^{\star \star *}$ & $(-3,32)$ & $-0,21$ & $(-1,38)$ & $-0,21^{*}$ & $(-1,931)$ & $-0,20$ * & $(-2,02)$ \\
\hline PRA_VALADO/SAINTVIT & $-0,28^{\star \star}$ & $(-2,28)$ & $-0,19^{*}$ & $(-1,67)$ & $-0,36^{\star \star \star}$ & $(-3,453)$ & $-0,34^{\star \star \star}$ & $(-3,35)$ \\
\hline PRA_MONTAG & $-0,03$ & $(-0,14)$ & $-0,14$ & $(-0,62)$ & - & - & - & - \\
\hline LNSAU & $-0,06$ & $(-1,05)$ & $-0,02$ & $(-0,43)$ & $-0,01$ & $(-0,16)$ & $-0,01$ & $(-0,10)$ \\
\hline PSAU_FOURR & $-0,29$ & $(-1,35)$ & $-0,19$ & $(-0,98)$ & $-0,00$ & $(-1,192)$ & $-0,00$ & $(-1,19)$ \\
\hline NB_LABELS & 0,01 & $(0,4)$ & 0,02 & $(0,55)$ & $-0,03$ & $(-0,628)$ & $-0,03$ & $(-0,58)$ \\
\hline ALTMEAN & $-0,00$ & $(-0,1)$ & 0,00 & $(0,18)$ & - & - & - & - \\
\hline P_IRRIG & - & - & - & - & $-0,00$ & $(-0,2)$ & $-0,00$ & $(-0,25)$ \\
\hline P_ZH & $4,67^{\star *}$ & $(2,25)$ & 1,80 & $(0,9)$ & 0,00 & $(0,337)$ & 0,00 & $(0,37)$ \\
\hline DENSNETTE & $-0,00$ & $(-0,56)$ & $-0,00$ & $(-0,37)$ & $0,00^{\star \star *}$ & $(2,774)$ & $0,00^{\star *}$ & $(3,10)$ \\
\hline D_CFREQ & $-0,00$ & $(-0,21)$ & 0,00 & $(0,26)$ & $-0,00$ & $(-0,101)$ & $-0,00$ & $(-0,14)$ \\
\hline D_LIT & $-0,03^{\star \star \star}$ & $(-5,54)$ & $-0,36^{\star \star \star}$ & $(-3,69)$ & $-0,01^{\star \star \star}$ & $(-3,03)$ & $-0,01^{\star *}$ & $(-2,8)$ \\
\hline BP1 & - & - & $0,34^{\star \star *}$ & $(3,56)$ & - & - & - & - \\
\hline BP2 & - & - & - & - & - & - & - & - \\
\hline$\lambda$ & & & -0 & & & & & \\
\hline Test de Hausman spatial (proba) & & & 36,18 & $(0,14)$ & & & 32,09 & $(0,16)$ \\
\hline $\mathrm{N}$ & & & & & & & & \\
\hline $\mathrm{R}^{2}$ ajusté & & & & & & & & \\
\hline 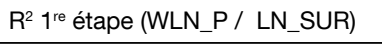 & & & 0,73 & 0,86 & & & 0,38 & 0,79 \\
\hline I de Moran - VI (probabilité) & & & $-1,41$ & $(0,16)$ & & & $-0,93$ & $(0,35)$ \\
\hline Test $J$ de Hansen (probabilité) & & & 75,46 & $(0,03)$ & & & 42,37 & $(0,70)$ \\
\hline Test $C$ d'endogénéité (probabilité) & & & 1,36 & $(0,24)$ & & & 0,043 & $(0,83)$ \\
\hline
\end{tabular}

Lecture : pour chaque segment de marché et pour chaque zone d'étude, les annexes 3, 4 et 5 présentent le modèle de référence ( $M C O$ »), le modèle de première étape de la procédure de Fingleton et Le Gallo (2008) ("IV-SAR », i.e. un modèle avec variable endogène décalée spatialement et instrumentée), et, en cas d'endogénéité, le modèle final " FGS2SLS », Feasible Generalized Spatial Two Stage Least Square) (cf. encadré 4). Les niveaux de risque des tests sont notés : 1 \% "***', 5 \% "**' et 10 \% "*'. Les statistiques de test (t de Student ou statistiques z pour les modèles estimés par les doubles moindres carrés) sont données entre parenthèses. Les variables de ce modèle sont les variables transformées selon la procédure de Cochrane-Orcutt.

Champ : transactions Safer échantillonnées par terrain d'étude, uniquement segment "Maisons à la Campagne ».

Source : traitement et calculs des auteurs d'après les données extraites des bases Safer. 
ANNEXE 4

Résultats des estimations sur le marché de l'artificialisation

\begin{tabular}{|c|c|c|c|c|c|c|c|c|c|c|c|c|}
\hline \multirow{3}{*}{$\begin{array}{c}\text { Variables } \\
\text { Constante }\end{array}$} & \multicolumn{6}{|c|}{ Côte basque } & \multicolumn{6}{|c|}{ Littoral charentais } \\
\hline & \multicolumn{2}{|c|}{ MCO } & \multicolumn{2}{|c|}{ IV-SAR } & \multicolumn{2}{|c|}{ FGS2SLS } & \multicolumn{2}{|c|}{ MCO } & \multicolumn{2}{|c|}{ IV-SAR } & \multicolumn{2}{|c|}{ FGS2SLS } \\
\hline & $13,33^{\star \star \star}$ & $(26,34)$ & $14,29^{\star \star \star}$ & $(9,06)$ & $13,28^{\star \star \star}$ & $(9,24)$ & $11,58^{\star \star \star}$ & $(29,784)$ & $10,9^{\star \star \star}$ & $(25,62)$ & $8,89^{\star \star \star}$ & $(15,08)$ \\
\hline WLN_P & - & & 0,084 & $(0,87)$ & $-0,007$ & $(-0,07)$ & - & - & 0,009 & $(0,18)$ & $-0,039$ & $(-0,75)$ \\
\hline X2002 & $-0,26^{*}$ & $(-1,79)$ & $-0,29^{*}$ & $(-1,84)$ & $-0,30^{*}$ & $(-1,81)$ & - & - & - & - & - & - \\
\hline X2003 & 0,09 & $(0,6)$ & 0,07 & $(0,42)$ & 0,04 & $(0,25)$ & Ref. & Ref. & Ref. & Ref. & Ref. & Ref. \\
\hline X2004 & $0,23^{\star}$ & $(1,73)$ & 0,19 & $(1,22)$ & 0,18 & $(1,14)$ & $0,17^{\star \star \star}$ & $(28,7)$ & $0,18^{\star \star \star}$ & $(3,87)$ & $0,18^{\star \star \star}$ & $(3,86)$ \\
\hline X2005 & $0,53^{\star \star \star}$ & $(3,91)$ & $0,47^{\star \star \star}$ & $(2,9)$ & $0,46^{\star \star \star}$ & $(2,78)$ & $0,47^{\star \star \star}$ & $(9,321)$ & $0,48^{\star \star \star}$ & $(10,36)$ & $0,48^{\star \star \star}$ & $(10,39)$ \\
\hline X2006 & $0,45^{\star \star \star}$ & $(3,42)$ & $0,41^{\star *}$ & $(2,42)$ & $0,40^{\star \star}$ & $(2,28)$ & $0,57^{\star \star \star}$ & $(11,256)$ & $0,57^{\star \star \star}$ & $(11,96)$ & $0,58^{\star \star \star}$ & $(11,74)$ \\
\hline X2007 & $0,57^{\star \star \star}$ & $(4,53)$ & $0,54^{\star \star \star}$ & $(3,29)$ & $0,53^{\star \star \star}$ & $(3,16)$ & - & - & - & - & - & - \\
\hline X2008 & $0,26^{\star}$ & $(1,9)$ & 0,22 & $(1,1)$ & 0,20 & $(1,09)$ & - & - & - & - & - & - \\
\hline LN_SUR & $0,55^{\star \star \star}$ & $(14,25)$ & $0,47^{\star \star \star}$ & $(9,22)$ & $0,48^{\star \star \star}$ & $(9,72)$ & $0,49^{\star \star \star}$ & $(29,78)$ & $0,45^{\star \star \star}$ & $(21,85)$ & $0,46^{\star \star \star}$ & $(22,25)$ \\
\hline BATI & $0,34^{*}$ & $(1,73)$ & 0,30 & $(1,08)$ & 0,35 & $(1,24)$ & $0,6^{\star \star \star}$ & $(5,923)$ & $0,60^{\star \star \star}$ & $(5,15)$ & $0,61^{\star \star \star}$ & $(5,31)$ \\
\hline $\begin{array}{l}\text { A_AQUI/ } \\
\text { CHARENTE }\end{array}$ & $-0,13$ & $(-1,24)$ & $-0,12$ & $(-1,38)$ & $-0,09$ & $(-1,07)$ & $-0,15^{\star \star \star}$ & $(-2,7)$ & $-0,15^{\star \star}$ & $(-3,27)$ & $-0,16^{\star \star \star}$ & $(-3,67)$ \\
\hline $\begin{array}{l}\text { V_AQUI/ } \\
\text { CHARENTE }\end{array}$ & 0,06 & $(0,57)$ & 0,03 & $(0,29)$ & 0,01 & $(0,15)$ & $-0,11^{*}$ & $(-1,866)$ & $-0,10^{*}$ & $(-1,75)$ & $-0,10^{*}$ & $(-1,80)$ \\
\hline V_PROFAGRI & $-0,22^{\star \star}$ & $(-2,22)$ & $-0,18^{\star}$ & $(-2,03)$ & $-0,18^{\star \star}$ & $(-2,03)$ & $-0,11^{*}$ & $(-1,982)$ & $-0,08$ & $(-1,31)$ & $-0,05$ & $(-0,70)$ \\
\hline A_PROFAGR & $-0,74^{\star \star}$ & $(-2,52)$ & $-0,81^{\star}$ & $(-1,74)$ & $-0,79^{\star}$ & $(-1,76)$ & $-0,4^{\star \star \star}$ & $(-3,6)$ & $-0,37^{\star \star \star}$ & $(-2,44)$ & $-0,35^{\star \star \star}$ & $(-2,35)$ \\
\hline V_ETABPUB & $-0,31^{\star \star}$ & $(-2,32)$ & $-0,28^{\star}$ & $(-1,65)$ & $-0,34^{\star}$ & $(-1,86)$ & $-0,03$ & $(-0,415)$ & $-0,07$ & $(-0,75)$ & $-0,12$ & $(-1,21)$ \\
\hline A_ETABPUB & $-0,56^{\star \star}$ & $(-2,06)$ & $-0,39$ & $(-1,19)$ & $-0,34$ & $(-1,02)$ & $-1,73^{\star \star \star}$ & $(-28,86)$ & $-1,68^{\star \star \star}$ & $(-17)$ & $-1,68^{\star \star \star}$ & $(-17,2)$ \\
\hline $\begin{array}{l}\text { PRA_MARENN/ } \\
\text { ROCH }\end{array}$ & $-0,84^{\star \star \star}$ & $(-3,6)$ & $-0,98^{\star \star \star}$ & $(-3,8)$ & $-1,04^{\star * \star}$ & $(-3,51)$ & $-0,71^{\star \star \star}$ & $(-7,292)$ & $-0,71^{\star \star *}$ & $(-5,79)$ & $-0,72^{\star \star \star}$ & $(-5,08)$ \\
\hline $\begin{array}{l}\text { PRA_GOSSE/ } \\
\text { POITEV }\end{array}$ & $-1,05^{\star \star \star}$ & $(-4,38)$ & $-1,24^{\star \star \star}$ & $(-3,27)$ & $-1,28^{\star \star \star}$ & $(-2,99)$ & $-0,003$ & $(-0,035)$ & $-0,1$ & $(-1,20)$ & $-0,1$ & $(-0,97)$ \\
\hline $\begin{array}{l}\text { PRA_SEIGNA } \\
\text { SAINTA }\end{array}$ & $-0,48^{\star \star}$ & $(-2,25)$ & $-0,56^{\star \star}$ & $(-2,52)$ & $-0,64^{\star \star}$ & $(-2,45)$ & $-0,51^{\star \star \star}$ & $(-6,11)$ & $-0,41^{\star \star \star}$ & $(-4,22)$ & $-0,42^{\star \star \star}$ & $(-3,59)$ \\
\hline $\begin{array}{l}\text { PRA_VALADO/ } \\
\text { SAINTVI }\end{array}$ & $-0,38^{\star \star \star}$ & $(-3,4)$ & $-0,39^{\star \star \star}$ & $(-3,63)$ & $-0,42^{\star \star \star}$ & $(-3,4)$ & $-0,55^{\star \star \star}$ & $(-7,092)$ & $-0,55^{\star \star \star}$ & $(-5,90)$ & $-0,56^{\star \star \star}$ & $(-5,06)$ \\
\hline PRA_MONTAG & $0,66^{\star *}$ & $(2,38)$ & $0,81^{\star \star \star}$ & $(4,06)$ & $0,91^{\star \star \star}$ & $(3,85)$ & - & - & - & - & - & \\
\hline LNSAU & $-0,11^{*}$ & $(-1,68)$ & $-0,14^{\star \star}$ & $(-2,31)$ & $-0,16^{\star \star}$ & $(-2,32)$ & $-0,04$ & $(-0,778)$ & 0,007 & $(0,12)$ & 0,01 & $(0,18)$ \\
\hline PSAU_FOURR & $-0,39$ & $(-1,57)$ & $-0,52$ & $(-1,61)$ & $-0,54$ & $(-1,45)$ & $0,005^{\star \star \star}$ & $(3,8)$ & $0,004^{\star \star \star}$ & $(2,96)$ & $0,004^{\star \star *}$ & $(2,82)$ \\
\hline NB_LABELS & $-0,04$ & $(-1,08)$ & $-0,04$ & $(-1,29)$ & $-0,05$ & $(-1,31)$ & 0,05 & $(1,613)$ & $0,08^{\star \star \star}$ & $(2,44)$ & $0,09^{\star \star \star}$ & $(2,32)$ \\
\hline ALTMEAN & $-0,001^{*}$ & $(-1,84)$ & $-0,0015^{\star}$ & $(-1,86)$ & $-0,0017^{\star}$ & $(-1,79)$ & - & & - & & - & \\
\hline P_IRRIG & - & - & - & - & - & - & $-0,002$ & $(-1,50)$ & $-3,6 e-5$ & $(-0,02)$ & $-5,8 e-5$ & $(-0,03)$ \\
\hline P_ZH & 2,25 & $(0,86)$ & 3,07 & $(0,87)$ & 2,96 & $(0,75)$ & $-0,001$ & $(-0,26)$ & $-0,002$ & $(-0,48)$ & $-0,003$ & $(-0,56)$ \\
\hline DENSNETTE & $-0,0001$ & $(-1)$ & $-0,0035$ & $(-0,62)$ & $-0,0037$ & $(-0,55)$ & $0,0007^{\star \star \star}$ & $(6,26)$ & $0,0006^{* \star *}$ & $(3,55)$ & $0,0007^{\star * *}$ & $(3,29)$ \\
\hline D_CFREQ & 0,0006 & $(0,13)$ & $-0,0001$ & $(-0,5)$ & $-0,0001$ & $(-0,43)$ & $-0,009^{\star \star \star}$ & $(-3,52)$ & $-0,003$ & $(-1,08)$ & $-0,003$ & $(-0,98)$ \\
\hline D_LIT & $-0,04^{\star \star \star}$ & $(-5,92)$ & $-2,86^{\star \star \star}$ & $(-3,95)$ & $-2,95^{\star \star \star}$ & $(-3,88)$ & $-0,02^{\star \star \star}$ & $(-10,51)$ & 0,08 & $(1,08)$ & 0,09 & $(1,04)$ \\
\hline BP1 & - & - & $2,87^{\star \star \star}$ & $(3,95)$ & $2,96^{\star \star \star}$ & $(3,88)$ & - & - & $-0,11^{*}$ & $(-1,59)$ & $-0,12^{\star}$ & $(-1,49)$ \\
\hline BP2 & - & - & $-0,068^{\star \star \star}$ & $(-3,18)$ & $-0,07^{\star \star \star}$ & $(-2,87)$ & - & - & $0,13^{\star \star \star}$ & $(5,73)$ & $0,14^{\star \star \star}$ & $(5,15)$ \\
\hline BP3 & - & - & - & - & - & - & - & - & $-0,12^{\star \star \star}$ & $(-4,91)$ & $-0,13^{\star \star \star}$ & $(-4,35)$ \\
\hline$\lambda$ & & & 0,1 & & & & & & & & & \\
\hline $\begin{array}{l}\text { Test de } \\
\text { Hausman } \\
\text { spatial (proba) }\end{array}$ & & & 58,30 & $(0,003)$ & & & & & 57,01 & $(6 e-4)$ & & \\
\hline $\mathrm{N}$ & & & & & & & 27 & & & & & \\
\hline $\mathrm{R}^{2}$ ajusté & 0,4 & & & & 0,4 & & 0,4 & & & & & \\
\hline $\begin{array}{l}R^{2} 1^{\text {re éta- }} \\
\text { pe (WLN_P / } \\
\text { LN_SUR) }\end{array}$ & & & 0,65 & ),78 & 0,63 & ,76 & & & 0,38 &, 75 & 0,39 &, 75 \\
\hline
\end{tabular}


Résultats des estimations sur le marché de l'artificialisation (suite)

\begin{tabular}{|c|c|c|c|c|c|c|c|c|c|c|}
\hline \multirow{2}{*}{ Variables } & \multicolumn{5}{|c|}{ Côte basque } & \multicolumn{5}{|c|}{ Littoral charentais } \\
\hline & $\mathrm{MCO}$ & & & & SLS & $\mathrm{MCO}$ & & & FG & SLS \\
\hline (probabilité) & & 2,51 & $(0,0121)$ & 0,02 & $(0,9864)$ & & 2,36 & $(0,0181)$ & 0,33 & $(0,7416)$ \\
\hline $\begin{array}{l}\text { Test } J \text { de } \\
\text { Hansen } \\
\text { (probabilité) }\end{array}$ & & 68,96 & $(0,154)$ & 68,89 & $(0,155)$ & & 73,91 & $(0,0246)$ & 67,391 & $(0,0741)$ \\
\hline $\begin{array}{l}\text { Test } C \\
\text { d'endogénéité } \\
\text { (probabilité) }\end{array}$ & & 8,12 & $(0,004)$ & 8,47 & $(0,0036)$ & & 22,299 & $(0,000)$ & 21,039 & $(0,000)$ \\
\hline
\end{tabular}

Lecture : pour chaque segment de marché et pour chaque zone d'étude, les annexes 3,4 et 5 présentent le modèle de référence (« MCO »), le modèle de première étape de la procédure de Fingleton et Le Gallo (2008) ("IV-SAR », i.e. un modèle avec variable endogène décalée spatialement et instrumentée), et, en cas d'endogénéité, le modèle final ("FGS2SLS ", Feasible Generalized Spatial Two Stage Least Square) (cf. encadré 4). Les niveaux de risque des tests sont notés : $1 \%$ "***', 5 \% "**' et $10 \%$ "'. Les statistiques de test ( $t$ de Student ou statistiques z pour les modèles estimés par les doubles moindres carrés) sont données entre parenthèses. Les variables de ce modèle sont les variables transformées selon la procédure de Cochrane-Orcutt.

Champ : transactions Safer échantillonnées par terrain d'étude, uniquement segment "Artificialisation ».

Source : traitement et calculs des auteurs d'après les données extraites des bases Safer. 
ANNEXE 5

\section{Résultats des estimations sur le marché des terres et prés}

\begin{tabular}{|c|c|c|c|c|c|c|c|c|c|c|c|c|}
\hline \multirow{3}{*}{$\begin{array}{c}\text { Variables } \\
\text { Constante }\end{array}$} & \multicolumn{6}{|c|}{ Côte basque } & \multicolumn{6}{|c|}{ Littoral charentais } \\
\hline & \multicolumn{2}{|c|}{$\mathrm{MCO}$} & \multicolumn{2}{|c|}{ IV-SAR } & \multicolumn{2}{|c|}{ FGS2SLS } & \multicolumn{2}{|c|}{$\mathrm{MCO}$} & \multicolumn{2}{|c|}{ IV-SAR } & \multicolumn{2}{|c|}{ FGS2SLS } \\
\hline & $9,89^{\star \star \star}$ & $(10,22)$ & $8,18^{\star \star \star}$ & $(5,75)$ & $8,02^{\star \star \star}$ & $(5,72)$ & $9,44^{\star \star \star}$ & $(22,3)$ & $9,31^{\star \star \star}$ & $(17,32)$ & $8,57^{\star \star \star}$ & $(16,19)$ \\
\hline WLN_P & - & & 0,02 & $(0,44)$ & 0,02 & $(0,36)$ & - & & 0,015 & $(0,59)$ & 0,019 & $(0,73)$ \\
\hline X2002 & 0,32 & $(1,48)$ & 0,16 & $(0,86)$ & 0,15 & $(0,76)$ & - & - & - & - & - & - \\
\hline X2003 & 0,31 & $(1,46)$ & 0,28 & $(1,34)$ & 0,27 & $(1,32)$ & Ref. & Ref. & Ref. & Ref. & Ref. & Ref. \\
\hline X2004 & 0,25 & $(1,08)$ & 0,09 & $(0,41)$ & 0,08 & $(0,37)$ & 0,03 & $(0,69)$ & 0,04 & $(0,88)$ & 0,04 & $(0,87)$ \\
\hline X2005 & 0,005 & $(0,02)$ & $-0,07$ & $(-0,33)$ & $-0,08$ & $(-0,34)$ & 0,05 & $(1,03)$ & 0,06 & $(1,27)$ & 0,06 & $(1,24)$ \\
\hline X2006 & 0,18 & $(0,74)$ & 0,02 & $(0,09)$ & $-0,01$ & $(-0,02)$ & 0,03 & $(0,70)$ & 0,03 & $(0,67)$ & 0,03 & $(0,76)$ \\
\hline X2007 & 0,23 & $(1,02)$ & 0,32 & $(1,51)$ & 0,32 & $(1,48)$ & - & - & - & - & - & - \\
\hline X2008 & 0,22 & $(0,97)$ & 0,44 & $(1,63)$ & 0,43 & $(1,6)$ & - & - & - & - & - & - \\
\hline LN_SUR & $0,83^{\star \star \star}$ & $(17,57)$ & $1,46^{\star \star *}$ & $(16,82)$ & $1,47^{\star \star \star}$ & $(16,81)$ & $0,76^{\star \star *}$ & $(63,04)$ & $0,80^{\star \star \star}$ & $(57,24)$ & $0,80^{\star \star *}$ & $(57,13)$ \\
\hline BATI & $1,65^{\star \star \star}$ & $(8,34)$ & $1,25^{\star \star \star}$ & $(4,36)$ & $1,25^{\star \star \star}$ & $(4,31)$ & 2,03 *** & $(27,62)$ & $2,01^{\star \star \star}$ & $(15,47)$ & $2,00^{\star \star *}$ & $(15,46)$ \\
\hline $\begin{array}{l}\text { A_AQUI/ } \\
\text { CHARENTE }\end{array}$ & 0,12 & $(0,41)$ & 0,32 & $(1,08)$ & 0,31 & $(1,04)$ & $-0,22^{\star \star}$ & $(-2,43)$ & $-0,22^{\star \star}$ & $(-1,45)$ & $-0,22^{\star \star}$ & $(-1,42)$ \\
\hline $\begin{array}{l}\text { V_AQUI/ } \\
\text { CHARENTE }\end{array}$ & 0,04 & $(0,25)$ & 0,07 & $(0,37)$ & 0,08 & $(0,41)$ & $-0,002$ & $(-0,05)$ & 0,004 & $(0,09)$ & 0,005 & $(0,11)$ \\
\hline V_ETABPUB & $-0,14$ & $(-0,29)$ & $-0,14$ & $(-0,93)$ & $-0,15$ & $(-0,94)$ & $-0,16$ & $(-1,03)$ & $-0,13$ & $(-0,65)$ & $-0,14$ & $(-0,68)$ \\
\hline A_ETABPUB & $0,74^{\star \star \star}$ & $(2,8)$ & $-0,12$ & $(-0,83)$ & $-0,12$ & $(-0,8)$ & 0,003 & $(-0,06)$ & $-0,03$ & $(-0,50)$ & $-0,03$ & $(-0,47)$ \\
\hline V_PROFAGRI & $-0,1$ & $(-0,76)$ & 0,10 & $(0,25)$ & 0,10 & $(0,26)$ & 0,05 & $(1,34)$ & 0,04 & $(1,13)$ & 0,04 & $(1,11)$ \\
\hline A_PROFAGR & $-0,43^{\star \star \star}$ & $(-3,51)$ & $0,66^{\star}$ & $(1,76)$ & $0,65^{\star}$ & $(1,73)$ & $-0,56^{\star \star \star}$ & $(-12,98)$ & $-0,55^{\star \star \star}$ & $(-9,18)$ & $-0,55^{\star \star \star}$ & $(-9,18)$ \\
\hline $\begin{array}{l}\text { PRA_ } \\
\text { MARENN/ } \\
\text { ROCHEF }\end{array}$ & $-1,34^{\star \star \star}$ & $(-3,73)$ & $-1,28^{\star \star \star}$ & $(-3)$ & $-1,27^{\star \star \star}$ & $(-2,94)$ & $-0,29^{\star \star \star}$ & $(-4,19)$ & $-0,29^{\star \star \star}$ & $(-3,50)$ & $-0,29^{\star \star \star}$ & $(-3,25)$ \\
\hline $\begin{array}{l}\text { PRA_GOSSE/ } \\
\text { POITEVIN }\end{array}$ & $-0,99^{\star \star \star}$ & $(-2,97)$ & $-1,21^{\star \star \star}$ & $(-3,34)$ & $-1,22^{\star \star \star}$ & $(-3,28)$ & $-0,26^{\star \star \star}$ & $(-3,65)$ & $-0,28^{\star \star \star}$ & $(-3,84)$ & $-0,28^{\star \star \star}$ & $(-3,57)$ \\
\hline $\begin{array}{l}\text { PRA_SEIGNA/ } \\
\text { SAINTAGR }\end{array}$ & $-1,01^{\star \star \star}$ & $(-3,03)$ & $-1,11^{\star *}$ & $(-2,51)$ & $-1,11^{\star *}$ & $(-2,46)$ & $-0,16^{\star *}$ & $(-2,25)$ & $-0,15^{\star \star}$ & $(-2,28)$ & $-0,15^{\star *}$ & $(-2,09)$ \\
\hline $\begin{array}{l}\text { PRA_VALADO/ } \\
\text { SAINTVIT }\end{array}$ & $-0,42^{*}$ & $(-1,8)$ & $-0,74^{\star \star \star}$ & $(-2,75)$ & $-0,75^{\star \star \star}$ & $(-2,72)$ & $-0,06$ & $(-0,92)$ & $-0,04$ & $(-0,60)$ & $-0,04$ & $(-0,54)$ \\
\hline PRA_MONTAG & $-0,76$ & $(-1,43)$ & $-0,71$ & $(-0,98)$ & $-0,74$ & $(-0,99)$ & - & - & - & - & - & - \\
\hline LNSAU & 0,13 & $(1,23)$ & 0,14 & $(1,34)$ & 0,15 & $(1,34)$ & $-0,05$ & $(-0,75)$ & $-0,04$ & $(-0,54)$ & $-0,04$ & $(-0,49)$ \\
\hline PSAU_FOURR & $-0,81^{*}$ & $(-1,83)$ & $-0,78^{*}$ & $(-1,68)$ & $-0,76$ & $(-1,61)$ & $-0,002$ & $(-1,55)$ & $-0,002$ & $(-1,02)$ & $-0,002$ & $(-0,93)$ \\
\hline NB_LABELS & 0,03 & $(0,47)$ & 0,09 & $(1,2)$ & 0,09 & $(1,19)$ & $-0,06^{\star \star}$ & $(-2,07)$ & $-0,06^{\star \star}$ & $(-2,01)$ & $-0,06^{\star \star}$ & $(-1,88)$ \\
\hline ALTMEAN & $-0,0004$ & $(-0,33)$ & 0,0005 & $(0,35)$ & 0,0004 & $(0,29)$ & - & - & - & - & - & - \\
\hline P_IRRIG & - & - & - & - & - & - & $0,006^{\star \star *}$ & $(4,27)$ & $0,006^{\star \star \star}$ & $(3,96)$ & $0,006^{\star \star \star}$ & $(3,70)$ \\
\hline P_ZH & 4,71 & $(1,14)$ & 6,16 & $(1,1)$ & 6,33 & $(1,12)$ & $-0,005^{\star}$ & $(-2,03)$ & $-0,006^{\star}$ & $(-1,64)$ & $-0,006^{*}$ & $(-1,52)$ \\
\hline DENSNETTE & $0,0007^{\star \star \star}$ & $(2,72)$ & $-0,007$ & $(-0,72)$ & $-0,007$ & $(-0,71)$ & $0,001^{\star \star \star}$ & $(7,77)$ & $0,001^{\star \star \star}$ & $(3,63)$ & $0,001^{\star \star \star}$ & $(3,25)$ \\
\hline D_CFREQ & $-0,008$ & $(-0,93)$ & 0,0006 & $(1,2)$ & 0,0006 & $(1,2)$ & 0,003 & $(1,42)$ & $0,005^{\star \star}$ & $(2,07)$ & $0,005^{\star \star}$ & $(1,93)$ \\
\hline D_LIT & $-0,030^{\star \star \star}$ & $(-3,2)$ & $-0,0328^{\star \star \star}$ & $(-2,82)$ & $-0,0327^{\star \star \star}$ & $(-2,75)$ & $-0,001$ & $(-0,72)$ & $-0,02^{\star \star *}$ & $(-2,60)$ & $-0,02^{\star \star \star}$ & $(-2,47)$ \\
\hline BP1 & - & - & - & - & - & - & - & - & $0,03^{\star * \star}$ & $(2,98)$ & $0,03^{\star \star \star}$ & $(2,83)$ \\
\hline BP2 & - & - & - & - & - & - & - & - & $-0,02^{\star \star}$ & $(-1,99)$ & $-0,02^{\star \star}$ & $(-1,87)$ \\
\hline$\lambda$ & & & 0,0 & 554 & & & & & & 665 & & \\
\hline $\begin{array}{l}\text { Test de } \\
\text { Hausman } \\
\text { spatial (proba) }\end{array}$ & & & 43,46 & $(0,04125)$ & & & & & 30,903 & $(0,232)$ & & \\
\hline $\mathrm{N}$ & & & & & 4 & & & & & & & \\
\hline$R^{2}$ ajusté & 0,6 & & 0,4 & & 0,4 & & & & & & & \\
\hline $\begin{array}{l}R^{2} 1^{\text {re }} \\
\text { étape(WLN_P / } \\
\text { LN_SUR) }\end{array}$ & & & 0,91 & 0,54 & 0,91 & 0,54 & & & 0,6 & 0,80 & 0,65 & 0,80 \\
\hline $\begin{array}{l}\text { I de Moran - VI } \\
\text { (probabilité) }\end{array}$ & & & 0,30 & $(0,7678)$ & 0,25 & $(0,7994)$ & & & 1,89 & $(0,0592)$ & $-0,25$ & $(0,8017)$ \\
\hline
\end{tabular}


Résultats des estimations sur le marché des terres et prés (suite)

\begin{tabular}{|c|c|c|c|c|c|c|c|c|c|c|}
\hline \multirow{2}{*}{ Variables } & \multicolumn{5}{|c|}{ Côte basque } & \multicolumn{5}{|c|}{ Littoral charentais } \\
\hline & $\mathrm{MCO}$ & & & & SLS & MCO & & & FG & SLS \\
\hline $\begin{array}{l}\text { Hansen } \\
\text { (probabilité) }\end{array}$ & & 69,065 & $(0,1128)$ & 66,598 & $(0,1571)$ & & 24,289 & $(0,5027)$ & 24,683 & $(0,4802)$ \\
\hline $\begin{array}{l}\text { Test } C \\
\text { d'endogénéité } \\
\text { (probabilité) }\end{array}$ & & 60,092 & $(0,000)$ & 62,950 & $(0,000)$ & & 37,963 & $(0,000)$ & 37,633 & $(0,000)$ \\
\hline
\end{tabular}

Lecture : pour chaque segment de marché et pour chaque zone d'étude, les annexes 3,4 et 5 présentent le modèle de référence ( $M C O$ "), le modèle de première étape de la procédure de Fingleton et Le Gallo (2008) («IV-SAR », i.e. un modèle avec variable endogène décalée spatialement et instrumentée), et, en cas d'endogénéité, le modèle final ("FGS2SLS ", Feasible Generalized Spatial Two Stage Least Square) (cf. encadré 4). Les niveaux de risque des tests sont notés : 1 \% "***', 5 \% "**' et $10 \%$ "*'. Les statistiques de test (t de Student ou statistiques z pour les modèles estimés par les doubles moindres carrés) sont données entre parenthèses. Les variables de ce modèle sont les variables transformées selon la procédure de Cochrane-Orcutt.

Champ : transactions Safer échantillonnées par terrain d'étude, uniquement segment "Terres et Prés ».

Source : traitement et calculs des auteurs d'après les données extraites des bases Safer. 